\title{
Characterization of the SKA1-Low prototype station Aperture Array Verification System 2
}

\author{
Giulia Macario $\odot,{ }^{\mathrm{a}, *}$ Giuseppe Pupillo $\odot,{ }^{\mathrm{b}}$ Gianni Bernardi $\odot$,, ,c,d \\ Pietro Bolli $\odot$, a Paola Di Ninni, ${ }^{a}$ Giovanni Comoretto $\odot,{ }^{a}$ Andrea Mattana $\odot$, \\ Jader Monari, ${ }^{\text {b }}$ Federico Perini $\odot$, ${ }^{\mathrm{b}}$ Marco Schiaffino, ${ }^{\mathrm{b}}$ Marcin Sokolowski $\odot$, \\ Randall Wayth $\odot,{ }^{\mathrm{e}}$ Jess Broderick $\odot,{ }^{\mathrm{e}}$ Mark Waterson, ${ }^{\mathrm{f}}$ Maria Grazia Labate, \\ Riccardo Chiello, ${ }^{\mathrm{g}}$ Alessio Magro ${ }^{\mathrm{h}}{ }^{\mathrm{h}}$ Tom Booler, ${ }^{\mathrm{e}}$ Andrew Mcphail, \\ Dave Minchin, ${ }^{\mathrm{e}}$ and Raunaq Bhushan ${ }^{\mathrm{e}}$ \\ aIstituto Nazionale di Astrofisica (INAF), Osservatorio Astrofisico di Arcetri, Firenze, Italy \\ ${ }^{\mathrm{b}}$ Istituto Nazionale di Astrofisica (INAF), Istituto di Radioastronomia, Bologna, Italy \\ ${ }^{c}$ Rhodes University, Department of Physics and Electronics, Makhanda, South Africa \\ ${ }^{\mathrm{d} S}$ South African Radio Astronomy Observatory, Cape Town, South Africa \\ ${ }^{e}$ Curtin University, International Centre for Radio Astronomy Research (ICRAR), Perth, \\ Western Australia, Australia

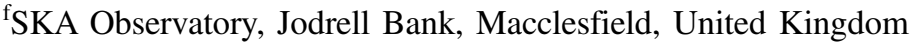 \\ ${ }^{g}$ University of Oxford, Oxford, United Kingdom \\ ${ }^{\text {h}}$ University of Malta, Institute of Space Sciences and Astronomy, Msida, Malta
}

\begin{abstract}
The low frequency component of the Square Kilometre Array (SKA1-Low) will be an aperture phased array located at the Murchison Radio-astronomy Observatory (MRO) site in Western Australia. It will be composed of 512 stations, each consisting of 256 log-periodic dual-polarized antennas, and will operate in the low frequency range (50 to $350 \mathrm{MHz}$ ) of the SKA bandwidth. The Aperture Array Verification System 2 (AAVS2), operational since late 2019, is the last full-size engineering prototype station deployed at the MRO site before the start of the SKA1-Low construction phase. The aim of this paper is to characterize the station performance through commissioning observations at six different frequencies $(55,70,110,160,230$, and $320 \mathrm{MHz}$ ) collected during its first year of activities. We describe the calibration procedure, present the resulting all-sky images and their analysis, and discuss the station calibratability and system stability. Using the difference imaging method, we also derive estimates of the SKA1-Low sensitivity for the same frequencies and compare them with those obtained through electromagnetic simulations across the entire telescope bandwidth, finding good agreement (within 13\%). Moreover, our estimates exceed the SKA1-Low requirements at all considered frequencies by up to a factor of $\sim 2.3$. Our results are very promising and allow for an initial validation of the AAVS2 prototype station performance, which is an important step toward the coming SKA1-Low telescope construction and science. () The Authors. Published by SPIE under a Creative Commons Attribution 4.0 International License. Distribution or reproduction of this work in whole or in part requires full attribution of the original publication, including its DOI. [DOI: 10.1117/1.JATIS.8.1.011014]
\end{abstract}

Keywords: radioastronomy; Square Kilometre Array; phased array telescopes; instrumentation.

Paper 21078SS received Jul. 30, 2021; accepted for publication Dec. 1, 2021; published online Jan. 5, 2022.

\section{Introduction}

The Square Kilometre Array (SKA) will be the world's largest and most sensitive radio telescope ever built, covering the huge fractional bandwidth from $50 \mathrm{MHz}$ to $15.3 \mathrm{GHz}$. It will bring a revolution in astronomy and astrophysics in the coming decades. ${ }^{1}$ When completed, it will consist of two telescopes: the SKA-Mid for the highest frequency end of the bandwidth (above $350 \mathrm{MHz}$, in the Karoo region of South Africa) and the SKA-Low for the lowest (below $350 \mathrm{MHz}$, in the Murchison Desert of Western Australia).

*Address all correspondence to Giulia Macario, giulia.macario@inaf.it 
The SKA-Low, to be built in the coming years at the Murchison Radio-astronomy Observatory (MRO) site, will operate with unprecedented sensitivity and resolution in the frequency range from 50 to $350 \mathrm{MHz}$. In its current design for phase 1, SKA1-Low will be an aperture phased array telescope consisting of 131,072 fixed dual-polarization log-periodic antennas, arranged in 512 stations of about 40 m diameter each, containing 256 antennas, ${ }^{1}$ with highly flexible digital beam-forming driven by advanced electronics. Almost $50 \%$ of the stations will be located in a very dense core of $\approx 1 \mathrm{~km}$ diameter, while the remaining stations will be distributed along three quasi-spiral arms, with a maximum baseline of $65 \mathrm{~km} .^{2}$

The deployment of prototype stations is a crucial step in the engineering design and development process before the construction of large and complex systems using new and/or unproven technology, such as SKA-Low. Since 2016, various full-size prototype SKA-Low stations have been deployed and tested at the MRO [e.g., Aperture Array Verification System 1 (AAVS1) ${ }^{3}$ and Engineering Development Array 1 (EDA1) ${ }^{4}$. In this paper, we will focus on the latest prototype station built at MRO, the AAVS2 $2^{5,6}$, deployed in 2019 and currently operational along with the comparator system EDA2 (see Ref. 7 submitted to this journal, for details). In the following sections, after a brief description of AAVS2 (Sec. 2), we present a first characterization of the array performance based on the most relevant results obtained through AAVS2 observations since its first light along with the simulations (Secs. 3 and 4.2).

\section{Background}

The AAVS2 is the most recent full-sized SKA-Low station prototype, based on the experience gained from the commissioning results of its predecessor AAVS1. ${ }^{3,6}$ It consists of 256 SKALA4.1 log-periodic dual-polarization antennas with low noise amplifiers optimized to meet the required performances in the SKA1-Low frequency bandwidth of 50 to $350 \mathrm{MHz} .{ }^{8}$ Antennas are pseudorandomly distributed over a circular area of about $40 \mathrm{~m}$ diameter, with a layout chosen to improve the overall performance of the station. ${ }^{6}$ The layout is basically identical to that of EDA2 and the predecessor stations, ${ }^{3,7}$ although the diameter of AAVS2 has been increased by $\sim 10 \%$ to host the larger SKALA4.1 antennas (the maximum distance among antennas is $\approx 38 \mathrm{~m}^{9}$ ).

A picture of the AAVS2 station is shown in Fig. 1 along with the station layout, showing the positions of the antennas with respect to the center of the array. Antennas are fixed to a wire ground mesh aligned with local cardinal directions [Fig. 1(a)], so antenna dipoles are oriented North-South and East-West. ${ }^{7}$ Coaxial cables connect antennas to 16 "SMART" boxes [one for each group of 16 antennas, Fig. 1(b)], each one converting radio frequency to optical signals. The signals, after being aggregated in a nearby field node distribution hub, are then transmitted

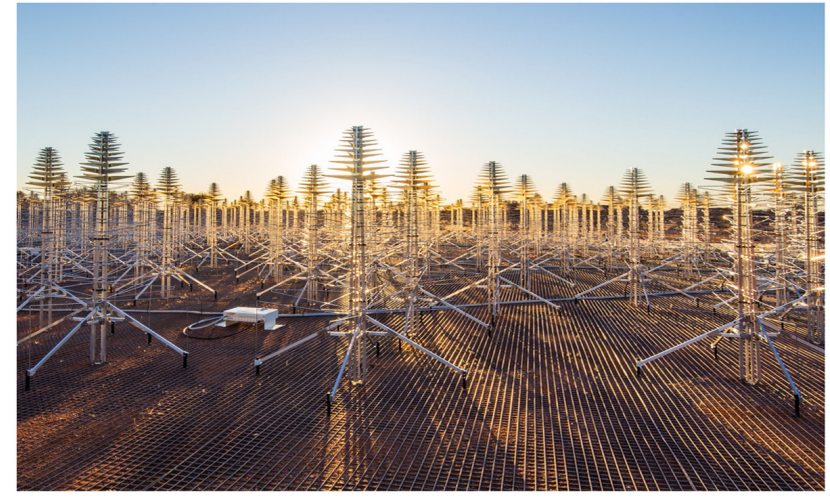

(a)

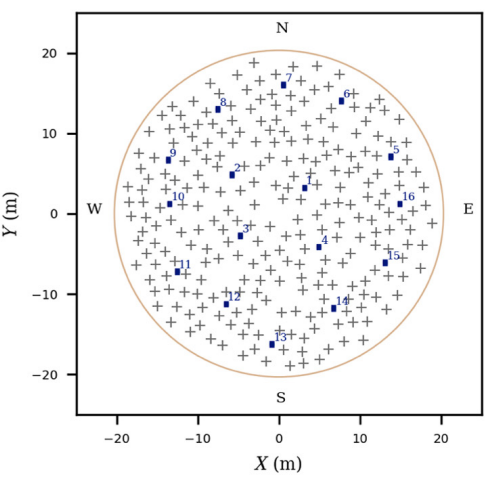

(b)

Fig. 1 (a) AAVS2 picture showing some of the SKALA4.1 aluminum log-periodic antennas. Credits: INAF/ICRAR. (b) AAVS2 array layout, showing positions of the 256 antennas (black crosses), pseudorandomly distributed within $\sim 40 \mathrm{~m}$ diameter area (yellow circle). The blue rectangles indicate the position of the 16 SMART boxes. 
through a $\sim 5.5 \mathrm{~km}$ optical fiber connection to the shielded room at the MRO control building, where the analog receivers and the acquisition and digital elaboration systems based on field programmable gate arrays are located. In the control room, optical signals are connected to 16 Tile Processing Modules (TPMs), where different data outputs are produced. A complete description of the AAVS2 system and signal chain is reported in Ref. 6 (their section 2 and figure 2). The SKA1-Low signal reception chain is broadly described in Ref. 10 (submitted to this issue). For detailed descriptions of the station monitoring and control software, observing modes, and data processing, we refer the reader to Refs. 3 (their sections 3.4 and 3.5) and 11 (their section 2).

AAVS2 has the same signal processing back-end and control elements as its predecessor AAVS1 (see Ref. 3). The two systems differ only in the station front-end design and architecture. In particular, beyond the replacement of SKALA2.0 with the SKALA4.1 antenna (and subsequent increase of the station diameter), AAVS2 now has a distributed approach of power and of the receiving analog signal, and the Front-End Modules (previously located at the apex of the antenna) were moved to a dedicated power and signal distribution box connecting each 16 antennas tile (see Ref. 6).

\section{Observations and Data Processing}

Observations with the AAVS2 station as a stand-alone interferometer were carried out at coarse channel central frequencies $\nu_{c}=54.7,70.3,110.2,159.4,229.8$, and $320.3 \mathrm{MHz}$ to commission and test the array. These frequencies were chosen to approximately match the frequencies used to specify SKA1-Low requirements and provide a reasonable number of sampling points across the bandwidth (see Refs. 7, 9, and 11, submitted to this issue). The exact frequency values were also chosen to avoid known satellite and terrestrial radio frequency interference (RFI) at the MRO (see Ref. 12, submitted to this issue, and Ref. 7). Throughout the paper, we will refer to these central frequencies as 55, 70,110, 160, 230, and $320 \mathrm{MHz}$, for simplicity. Data were taken in April 2020 apart from the $54.7 \mathrm{MHz}$ frequency that was observed on February 19, 2021, all of them spanning a time interval of at least $22 \mathrm{~h}$ (Table 1). Observations consisted of a series of $0.28 \mathrm{~s}$ snapshots (separated into two $0.14 \mathrm{~s}$ integrations) repeated every $5 \mathrm{~min}$, using a single $925.926 \mathrm{kHz}$ wide coarse channel. There was no evidence of significant RFI in any observation, except for two snapshots at $230 \mathrm{MHz}$ affected by interference from satellite emission (on April 22, 2020, between 16:45 and 16:55 UT), which were discarded from the analysis.

Visibilities were generated from the complex voltages of individual antennas using a software correlator, ${ }^{9,13}$ yielding 32 finer channels, each $28.935 \mathrm{kHz}$ wide. They were stored using the UVFITS format. ${ }^{14}$ At each observed frequency, the total number of visibilities recorded in each 0.28 s snapshot was 84,212,376 (all four polarizations and autocorrelations included). Delays due to the propagation along different cable lengths were calibrated using observations of the Sun (similar to the procedure described below) and then stored in lookup tables. As delays are

Table 1 Summary of AAVS2 observations used in this work.

\begin{tabular}{lccc}
\hline \hline $\begin{array}{l}\nu_{c} \\
(\mathrm{MHz})\end{array}$ & $\begin{array}{c}\text { Start time, UT } \\
\text { (yyyy/mm/dd, hh:mm:ss) }\end{array}$ & $\begin{array}{c}\text { LST coverage } \\
(\mathrm{h})\end{array}$ & $\begin{array}{c}\text { UT at Sun transit } \\
\text { (yyyy/mm/dd, hh:mm:ss) }\end{array}$ \\
\hline 54.7 & $2021 / 02 / 19,03: 03: 01$ & $\simeq 22$ & $2021 / 02 / 19,04: 27: 58$ \\
70.3 & $2020 / 04 / 17,08: 59: 25$ & $\simeq 24$ & $2020 / 04 / 18,04: 13: 44$ \\
110.2 & $2020 / 04 / 21,10: 19: 31$ & $\simeq 24$ & $2020 / 04 / 22,04: 13: 53$ \\
159.4 & $2020 / 04 / 07,16: 26: 58$ & $\simeq 24$ & $2020 / 04 / 08,04: 16: 33$ \\
229.8 & $2020 / 04 / 19,03: 52: 11$ & $\simeq 22$ & $2020 / 04 / 19,04: 12: 10$ \\
320.3 & $2020 / 04 / 22,11: 09: 11$ & $\simeq 24$ & $2020 / 04 / 23,04: 13: 34$ \\
\hline \hline
\end{tabular}


found to be stable over time scales of weeks, ${ }^{3}$ in our analysis we did not solve for delays but directly corrected for them using values in the lookup tables. ${ }^{3,7}$

Data processing was carried out with the Miriad package. ${ }^{15}$ Six and 12 malfunctioning antennas were permanently flagged in the April 2020 and February 2021 data, respectively. Five edge fine channels (the first three and the last two) were flagged too, to avoid band edges, reducing the coarse channel bandwidth to $\approx 780 \mathrm{kHz}$. For the purposes of our analysis, autocorrelations were also removed, and only the two linear polarizations $(X X$ and $Y Y)$ were used in the processing. Thus, the fraction of visibilities used for imaging each $0.28 \mathrm{~s}$ snapshot was $\approx 40 \%$ and $\approx 36 \%$ of the total visibilites recorded for April 2020 and February 2021 observations, respectively.

Figure 2 shows the $u v$-coverage and the corresponding synthesized beams (or point spread functions) for three snapshots at solar transit. As all snapshots are phased centered to zenith, $u v$ coverages scale by a factor of $\approx 3$ between 70 and $230 \mathrm{MHz}$. Conversely, synthesized beam sizes decrease going from low to high frequencies.

The Sun is unresolved at all frequencies by the longest baselines of the array and was therefore used as a point-like calibration source (similarly to previous works ${ }^{3,6,11}$ ). During our observations, there was no sign of solar activity (see Ref. 16). The low frequency solar spectrum is well measured down to $30 \mathrm{MHz}{ }^{17}$ In the 50 to $350 \mathrm{MHz}$ SKA1-Low frequency range, we approximated it with a power-law model with spectral index $\alpha_{i}$ changing with the frequency interval

$$
S_{\nu_{c}}=S_{\nu_{i}}\left(\frac{\nu_{c}}{\nu_{i}}\right)^{\alpha_{i}}
$$

where $\nu_{i}$ and $S_{\nu_{i}}$ are measurements from observations of the quiet $\operatorname{Sun}^{17}$ (first two columns of Table 2) and $\alpha_{i}$ is computed between the two $\nu_{i}$ values closest to each central frequency $\nu_{c}$ (third, fourth, and fifth columns of Table 2). The model flux densities of the Sun derived with Eq. (1) are
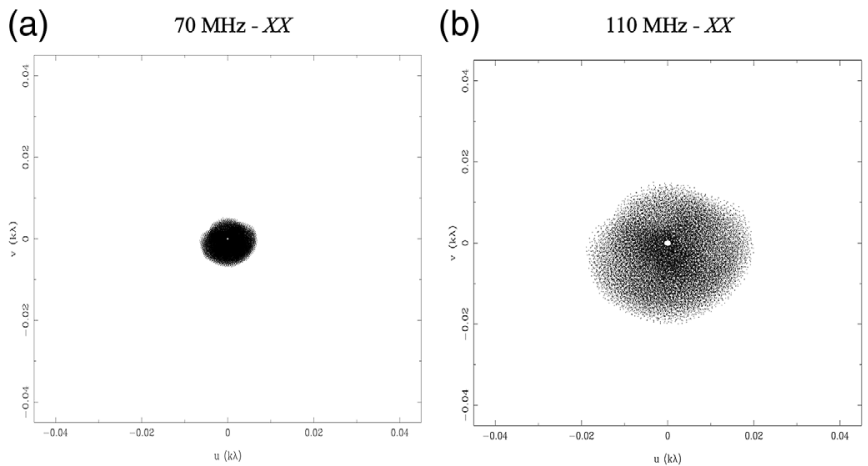

(c) $\quad 230 \mathrm{MHz}-X X$

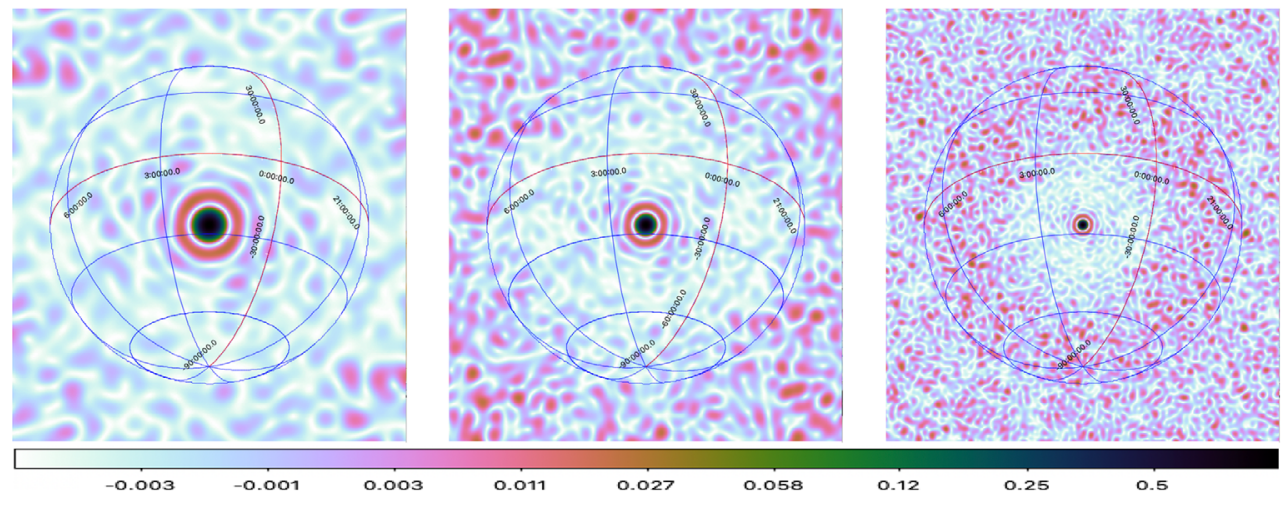

Fig. 2 AAVS2 uv-coverages (top row) and corresponding synthesized beams (bottom row) at $70 \mathrm{MHz}(\mathrm{a}), 110 \mathrm{MHz}$ (b), and $230 \mathrm{MHz}$ (c), corresponding to solar transit snapshots (see Table 1, last column). Only the $X X$ polarization is shown, with the $Y Y$ polarization being the same. All $u v$-coverages are plotted on the same $u, v$ scales $([-0.045,0.045] \mathrm{k} \lambda)$, and all dirty beams are on the same color scale (logarithmic, spanning the $[-0.005,1]$ range). 
Table 2 Power-law model flux densities of the Sun at the central frequencies of each observation $\left(S_{\nu_{c}}\right.$ and $\nu_{c}$, fifth and sixth columns, see Eq. (1)). These were derived from the Benz measurements ${ }^{17}\left(S_{\nu_{i}}\right.$ and $\nu_{i}$, first two columns, see Eq. (1)) using the spectral index values $\alpha_{i}$ (fourth column). These were computed in the Benz frequency interval closest to the central frequency of each observation ( $\Delta \nu$, third column). The last two columns are the expected SNR (per polarization) of the Sun close to its transit per baseline.

\begin{tabular}{lccccccc}
\hline \hline$\nu_{i}{ }^{17}(\mathrm{MHz})$ & $S_{\nu_{i}}{ }^{17}(\mathrm{Jy})$ & $\Delta \nu(\mathrm{MHz})$ & $\alpha_{i}$ & $\nu_{c}(\mathrm{MHz})$ & $S_{\nu_{c}}(\mathrm{Jy})$ & $\mathrm{SNR}_{X X}$ & $\mathrm{SNR}_{Y Y}$ \\
\hline 50 & 5400 & 50 to 100 & 2.15 & 54.7 & 6550 & 0.6 & 0.5 \\
& & & & 70.3 & 11200 & 1.6 & 1.4 \\
100 & 24,000 & 100 to 150 & 1.86 & 110.2 & 28700 & 12.6 & 7.8 \\
150 & 51,000 & 150 to 200 & 1.61 & 159.4 & 56200 & 31.4 & 19.9 \\
200 & 81,000 & 200 to 300 & 1.50 & 229.8 & 99800 & 58.5 & 28.6 \\
300 & 149,000 & 300 to 400 & 1.31 & 320.3 & 162000 & 68.9 & 32.7 \\
\hline \hline
\end{tabular}

reported in the sixth column of Table 2. The last two columns of Table 2 report the expected signal-to-noise ratios of the Sun at its transit per baseline, per linear polarization, at each observed frequency.

Calibration proceeded in the same way for each frequency: for the snaphots in which the Sun was closer to its transit (last column of Table 1), visibilities were rotated to the Sun position (by means of the task UVEDIT), and the complex bandpass was derived for each $0.14 \mathrm{~s}$ integration independently using the MFCAL task with a $0.14 \mathrm{~s}$ solution interval. To minimize the contribution from the Galactic emission, baselines $\leq 2 \lambda$ at frequencies $\leq 70 \mathrm{MHz}$ and $\leq 5 \lambda$ at the other higher frequencies were excluded from the calibration. This selection excluded from calibration the following fractions of baselines (for each snapshot and polarization): $\approx 20 \%$ at 70 and $160 \mathrm{MHz}, \approx 40 \%$ at $110 \mathrm{MHz}, \approx 12 \%$ at $230 \mathrm{MHz}$, and $\approx 6 \%$ at $320 \mathrm{MHz}$. Figure 3 shows calibration solutions for a subset of antennas. Solutions are approximately as expected: after delay correction, phase solutions are small $\left(10^{\circ} \max \right)$ and approximately zero-mean distributed. Phase

(a)

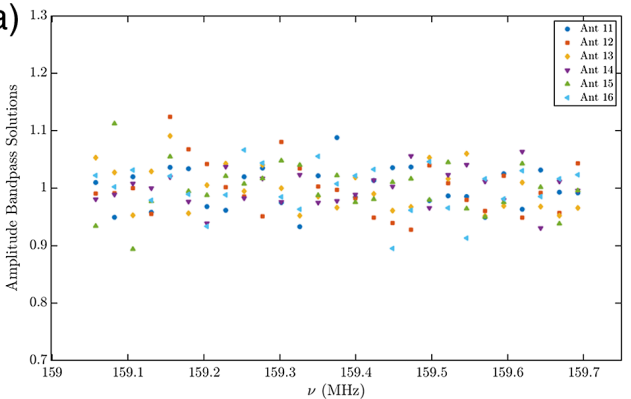

(b)

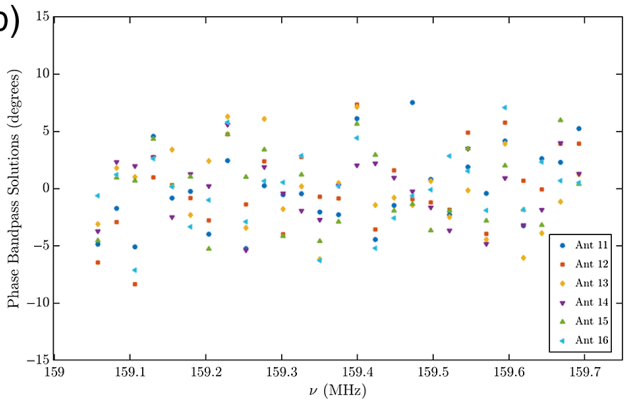

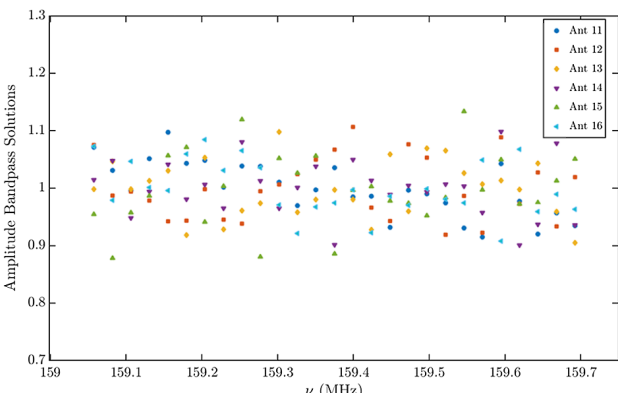

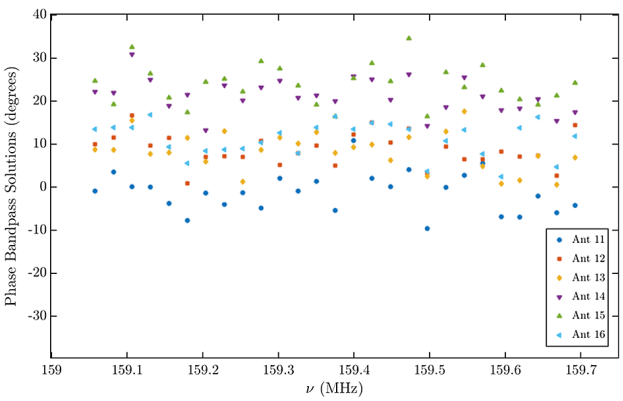

Fig. 3 Bandpass solutions for a subset of antennas: $X X(Y Y)$ polarization is displayed on the left (right), amplitude in (a), and phase in (b). 
Table 3 Average synthesized beams of AAVS2 all-sky images, for the analyzed frequencies (see Sec. 3 and Fig. 5). $\theta_{\text {maj }}$ and $\theta_{\text {min }}$ are the major and minor beam axes in degrees, and P.A. is the position angle (in degrees) measured from East to North.

\begin{tabular}{lc}
\hline \hline$\nu(\mathrm{MHz})$ & $\theta_{\text {maj }} \times \theta_{\text {min }}$, P.A. $\left({ }^{\circ} \times{ }^{\circ},{ }^{\circ}\right)$ \\
\hline 55 & $8.0 \times 7.9,9.0$ \\
70 & $6.3 \times 6.1,-31.0$ \\
110 & $4.0 \times 3.9,-31.1$ \\
160 & $2.8 \times 2.7,-31.1$ \\
230 & $1.9 \times 1.9,-31.0$ \\
320 & $1.4 \times 1.3,-31.1$ \\
\hline \hline
\end{tabular}

solutions for the $Y Y$ polarization display a $\sim 10^{\circ}$ offset from zero, probably due to a residual, uncalibrated delay-although further observations are needed to shed light on this issue. Bandpass amplitudes have a unity average, as expected, as MFCAL splits the calibration solutions between an overall amplitude across the bandwidth and the amplitude variations with respect to the mean (plotted in Fig. 3). Deviations from unity are within 10\%.

Calibration solutions obtained from the snapshot corresponding to the solar transit were transferred (through the GPCOPY task) to all other snapshots. Sun-based calibration failed at $55 \mathrm{MHz}$, where the solar emission is too faint with respect to the Galactic background emission (see Table 2, last two columns). To set the absolute flux density scale at this frequency, the procedure described in Sec. 3.1 was applied.

The antenna primary beam response was not used directly in the calibration model. Instead, we fixed the absolute flux density scale by multiplying each flux density and noise measurement of our analysis (see Secs. 3.1, 4.1, and 4.2) by the primary beam response corresponding to the Sun position in the snapshot observation used for calibration, normalized to zenith. We assumed that all antennas have the same primary beam, the average embedded element pattern $\left(\mathrm{EEP}^{5,18}\right)$. Examples of primary beams at each frequency are reported in Appendix A (Fig. 11).

Visibilities were Fourier transformed into zenith phase-centered all-sky images, using the MIRIAD task INVERT, with natural weighting and a pixel size of 21 arcmin — corresponding to 4 to 23 pixels across the synthesized beam, depending on the observing frequencies. For every snapshot across the entire observing window, each polarization was imaged separately, generating $X X$ and $Y Y$ images at each frequency. Unlike with calibration, no baseline was discarded in imaging. Each image covered the entire hemisphere visible at a specific time, with the synthesized beam ranging from $\approx 8.0^{\circ}$ to $\approx 1.4^{\circ}$ with increasing frequency (see Table 3 ). Dirty images were deconvolved using the Clark CLEAN algorithm with the Miriad CLEAN task, with a maximum of 200 iterations, to suppress sidelobes from the brightest sources and, simultaneously, avoiding over-cleaning. The quality of the obtained images remains good at all analyzed frequencies, with no evidence of phase and amplitude errors and an overall consistency of the morphology of the brightest sources (e.g., the Galactic plane and Centaurus A) across the $24 \mathrm{~h}$. In Sec. 4.1, we show that flux densities of bright, unresolved sources are consistent with the expected values $\approx 1 \mathrm{~h}$ across their transit, indicating that the system is stable over this time scale, within the measurement uncertainties.

Figure 4 shows images at 110 and $320 \mathrm{MHz}$ corresponding to the snapshot at solar transit (see last column of Table 1), obtained by averaging the two $0.14 \mathrm{~s}$ integrations of each snapshot (see Sec. 3). The Sun is clearly the dominant source in the sky, showing that the assumption of a point-like calibration model is appropriate.

Figure 5 shows examples of snapshot images at 110, 70, and $55 \mathrm{MHz}$, taken when Centaurus A, clearly visible at all frequencies, is approximately at transit $(\approx 12 \mathrm{~h}$ after solar culmination, i.e., calibration snapshot). Moreover, at the AAVS2 resolution, the Galactic plane is the brightest

J. Astron. Telesc. Instrum. Syst. $\quad$ 011014-6 Jan-Mar 2022 • Vol. 8(1) 


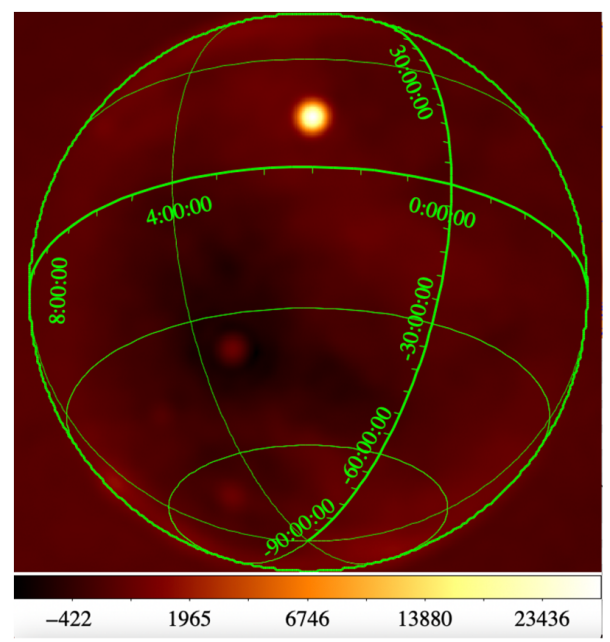

(a)

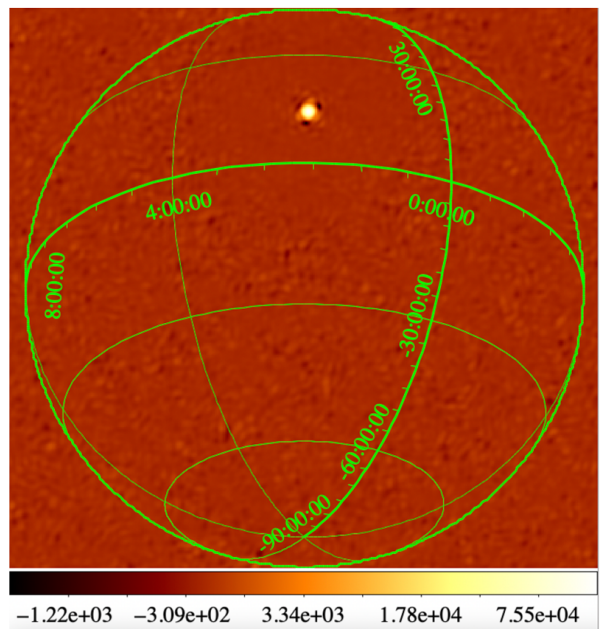

(b)

Fig. 4 All-sky, $X X$ polarization images at solar transit at 110 (a) and $320 \mathrm{MHz}$ (b). Units are Jy beam $^{-1}$.

visible feature, consistently detected across frequencies. Figure 5 also shows a comparison between simulated sky temperature images and actual observations.

The observable sky model at each frequency, i.e., the map of the sky brightness temperature above the horizon at a given time from the AAVS2 location, was derived from the global sky model (GSM), ${ }^{19}$ with contribution from the Sun emission added. ${ }^{17}$ The all-sky simulated maps were generated using the PyGSM code (see Ref. 20). For a better comparison with the actual observations, the sky model maps were smoothed to match the AAVS2 angular resolution at each frequency (see Table 3).

We note a qualitative good agreement between the observed and the simulated structures (upper and lower panels of Fig. 5, respectively), where Centaurus A is at the expected position. The Galactic plane has a more pronounced morphological difference, particularly at $55 \mathrm{MHz}$ for the $X X$ polarization and close to the horizon [Fig. 5(c), left side]. This discrepancy may be due to mutual coupling from the antennas, ${ }^{5,18}$ which is more prominent near the horizon and is not accounted for in our simulations. We also note that our simulated images do not include the station $u v$-coverage and that this can also cause slight differences between observed and simulated images, although a more quantitative comparison is left for the future. Note that the measured peak fluxes of Centaurus A (averaged between $X X$ and $Y Y$ polarizations) in the observed images (upper panels of Fig. 5), not corrected for the primary beam, are $\approx 8400, \approx 10,850$ and $\approx 11,000 \mathrm{Jy}$ at 110,70 , and $55 \mathrm{MHz}$, respectively, and the corresponding expected values (extrapolated from the literature measurements ${ }^{21}$ to each frequency) are $\approx 6950, \approx 9550$, and $\approx 11,300 \mathrm{Jy}$, respectively (see, however, Sec. 4.1 , for more details).

\subsection{Calibration at $55 \mathrm{MHz}$}

As mentioned in the previous section, the Sun is not sufficiently bright to serve as a calibration source at $55 \mathrm{MHz}$; therefore, we adopted the following strategy. All-sky snapshot images were produced from visibilities after applying delays only. Receiver gains were approximately equalized to have the same wide-band power at the TPM input for all antennas ${ }^{22}$; this first-order equalization, together with antenna-based delays, already allow for obtaining good images (Fig. 5 and Sec. 3).

The peak brightness values of the Sun and the corresponding uncertainties were extracted for the snapshots in the $21.7<\mathrm{LST}<22.7 \mathrm{~h}$ range $(\approx 1 \mathrm{~h}$ around transit $)$ using the IMFIT task, and corrected for the normalized mean $\mathrm{EEP}^{5,18}$ in the direction of the Sun. We found the apparent flux density of the Sun to be sufficiently constant in time, with variations within $\sim 15 \%$ (considering the average of both linear polarizations) across the selected local sidereal time (LST) range, that 


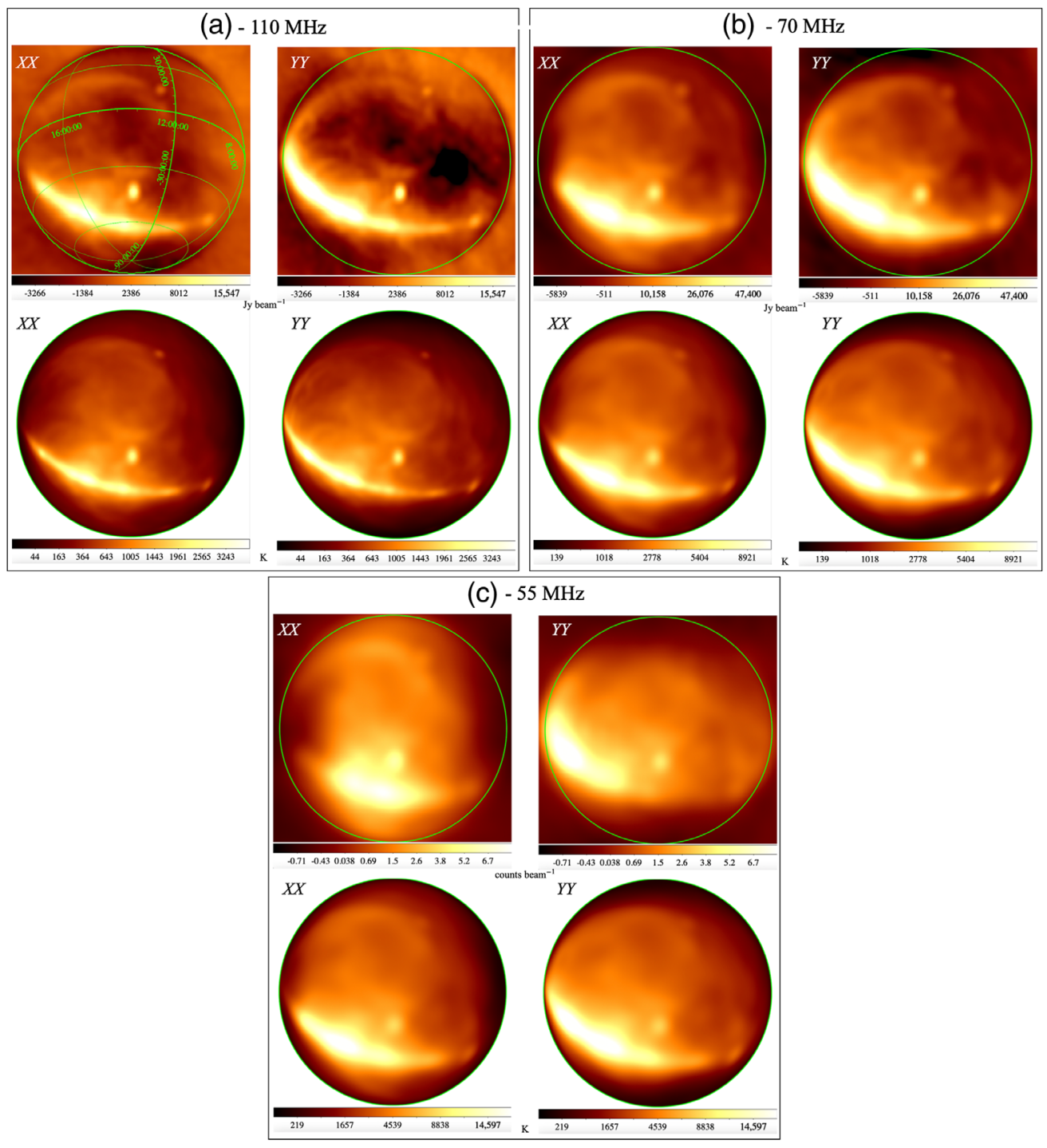

Fig. 5 All-sky, snapshot images at the transit of Centaurus $\mathrm{A}$, for $X X$ and $Y Y$ polarizations at 110 (a), 70 (b), and 55 (c). In each panel, the observed sky brightness (top frames) in Jy beam ${ }^{-1}$ (counts beam ${ }^{-1}$ for un-calibrated images at $55 \mathrm{MHz}$ ) is compared with the corresponding $T_{\text {sky }}$ simulations (bottom frames) in $\mathrm{K}$. Square root color scales are in the following ranges (for data and simulation, respectively): -3500 to $20,000 \mathrm{Jy} /$ beam, 4 to $4000 \mathrm{~K}$ at $110 \mathrm{MHz} ;-6500$ to $60,000 \mathrm{Jy} \mathrm{beam}^{-1}, 30$ to $11,000 \mathrm{~K}$ at $70 \mathrm{MHz} ;-0.8$ to 8.5 counts beam ${ }^{-1}, 40$ to $18,000 \mathrm{~K}$ at $55 \mathrm{MHz}$. The green circle indicates the horizon.

are within the uncertainties (see Fig. 6). This result shows that the simple power equalization does a reasonably good job in calibrating the visibility amplitudes.

We then calculated the ratio between the expected flux density of the Sun at $55 \mathrm{MHz}$ (Table 2) and the apparent flux density, averaged over the snapshots and corrected for the element primary beam per polarization. These scaling factors $(\approx 5600$ for $X X$ and $\approx 5200$ for $Y Y$ ) were used to bring our measurements at this frequency on the appropriate absolute flux density scale (Secs. 4.1 and 4.2). Results are shown in Fig. 6, where corrected solar flux densities with $1 \sigma$ error bars for each linear polarization are plotted against LST and compared with the Sun reference value at $55 \mathrm{MHz}$. To cross-check this procedure, we derived the (averaged and primary beam corrected) scaling factors per polarization using Centaurus A [see Sec. 4.1 and Fig. 7(a), for its reference flux value] within $\approx 1 \mathrm{~h}$ around its transit (LST range $\simeq 12.8$ to $13.9 \mathrm{~h}$ ), finding that they are within $\sim 10 \%$ of those derived from the Sun. 


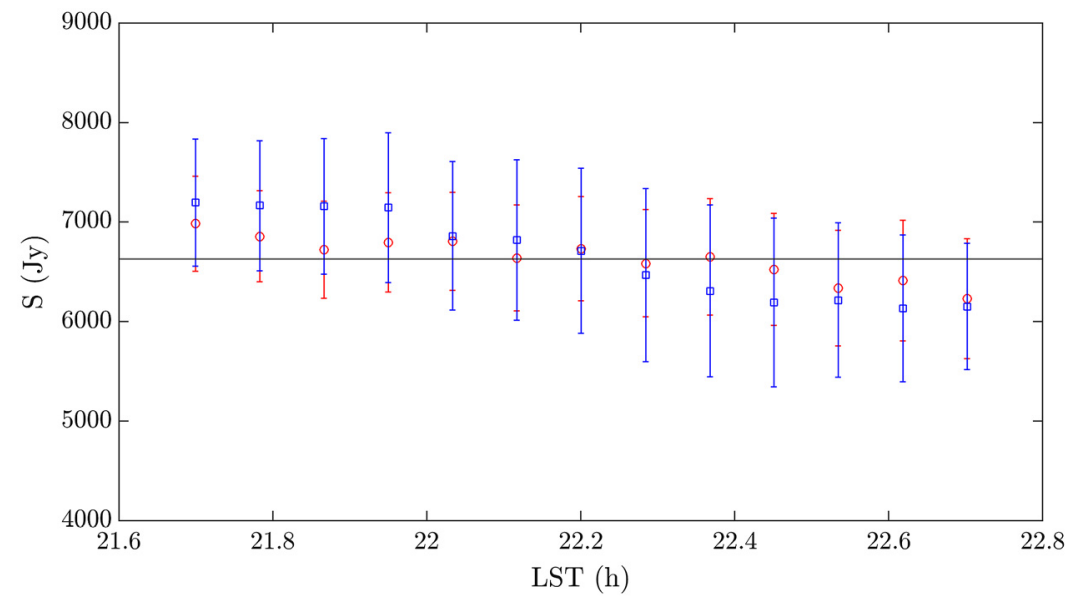

Fig. 6 Sun flux densities (in Jy) from uncalibrated snapshot images at $55 \mathrm{MHz}(\approx 1 \mathrm{~h}$ around Sun transit) with $1 \sigma$ error bars, after primary beam correction and a posteriori absolute flux scale calibration (Sec. 3.1), plotted as a function of LST (in h). Red and blue circles correspond to $X X$ and $Y Y$ polarization data, respectively. Solid line is the Sun reference flux density value (see Ref. 17 and Table 2).

(a)

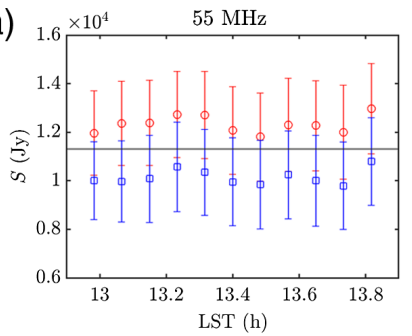

(b)

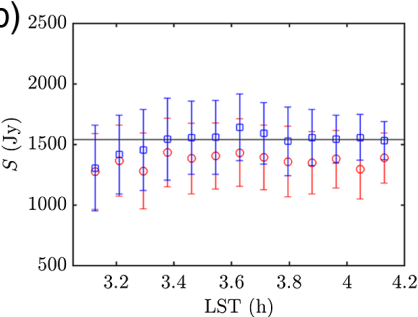

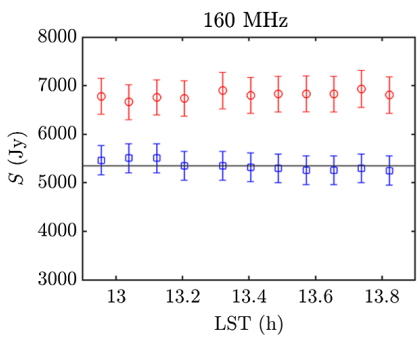
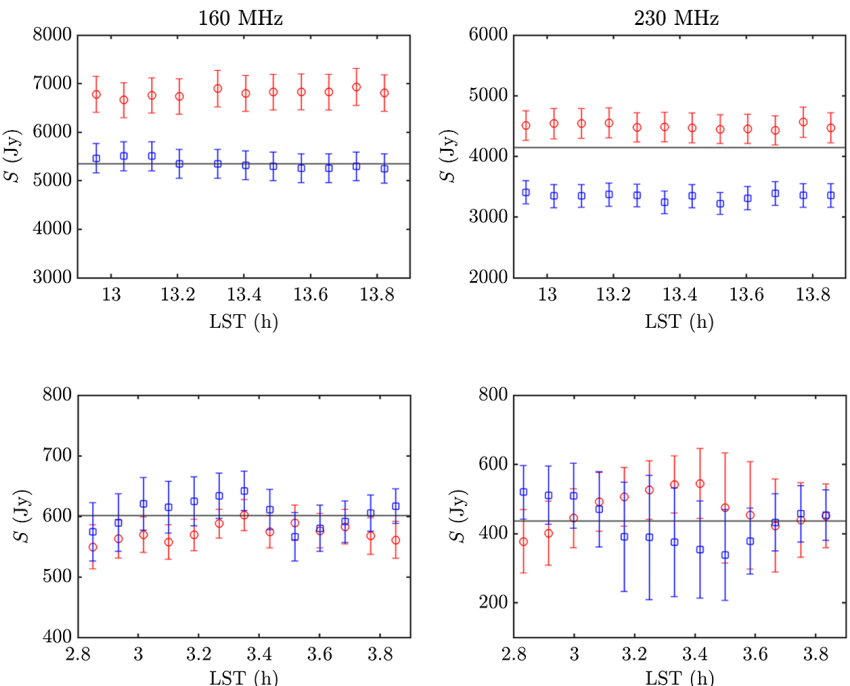

Fig. 7 (a) Centaurus $\mathrm{A}$ and (b) Fornax A flux densities (in Jy) with $1 \sigma$ error bars as a function of LST (in h), $\approx 1 \mathrm{~h}$ around their transit, for three selected frequencies $(55,160$, and $230 \mathrm{MHx}$, from left to right). Red circles and blue squares are the measurements from $X X$ and $Y Y$ polarization images, respectively. The black lines are the corresponding reference values, extrapolated from Refs. 21 and 23.

\section{Performances}

\subsection{Consistency Checks on Radio Sources Flux Densities}

To evaluate the time stability of calibration and assess the quality of the absolute flux scales, we performed the analysis of the flux densities of selected radio sources in the field (other than the Sun) as a function of time, and their consistency across the frequencies. Among the brightest "A-team" radio sources, we selected those with an elevation $\geq 60^{\circ}$ at their culmination. This criterion allows for analysis of the fluxes $\approx 1 \mathrm{~h}$ across the source transit, avoiding the sensitivity losses due to the poor SKALA4.1 antenna response at lower elevations (which is related to the natural drop off in the antenna design trade-off ${ }^{24}$ ). Only Centaurus A, Fornax A, and Pictor A met this selection criterion. We produced lightly cleaned all-sky images of snapshots in the 

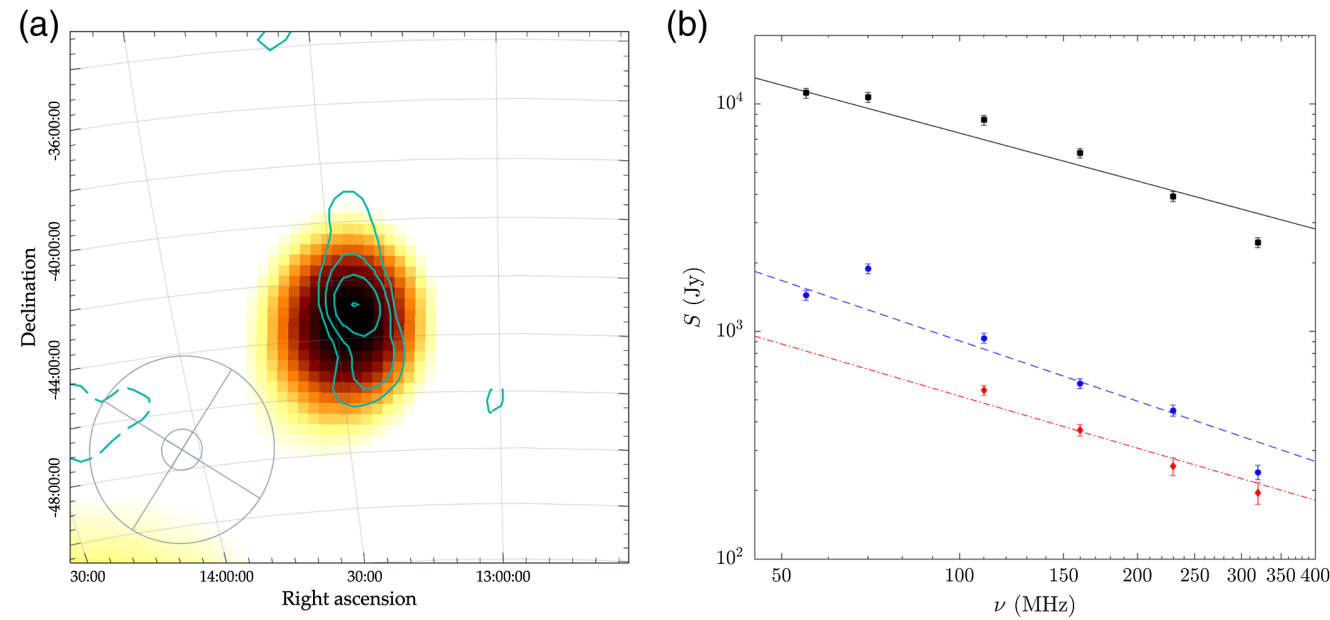

Fig. 8 (a) $70 \mathrm{MHz}$ image around Centaurus A, with the $320 \mathrm{MHz}$ contours overlaid. Both images are from $X X$ polarization, and include all baselines. The local $\sigma_{\mathrm{rms}}$ is $\simeq 1.3 \times 10^{4} \mathrm{Jy} \mathrm{beam}^{-1}$ at $70 \mathrm{MHz}$ and $\simeq 100 \mathrm{Jy}^{\text {beam }^{-1}}$ at $320 \mathrm{MHz}$. The color scale (linear) ranges from $2 \times 10^{4}$ to $3 \times 10^{4} \mathrm{Jy} \mathrm{beam}^{-1}$; cyan contours are spaced by a factor of two, starting from $\pm 2.5 \sigma_{\text {rms }}$ (dashed are negative). The beam sizes are shown by the gray circles (see Table 3 ). (b) Integrated spectra of Centaurus A (black squares), Fornax A (blue dots), and Pictor A (red diamonds). The lines are the power-law spectra from the literature, for a comparison: Centaurus A (black solid line, $\alpha=-0.70^{21}$ ), Fornax A (blue dashed line, $\alpha=-0.88^{23}$ ), and Pictor A (red dotted line, $\alpha=-0.76^{25}$ ).

selected LST ranges, with a similar procedure, as described in Sec. 3, but averaging the two $0.14 \mathrm{~s}$ integrations of each snapshot and excluding in the imaging the same baselines discarded in the calibration (see Sec. 3). The signal-to-noise ratios of Centaurus A and Fornax A are $\gtrsim 4-5$ at most of the frequencies, ensuring a good quality of the measured fluxes. However, Pictor A has a signal-to-noise ratio of $\lesssim 2.5$ at frequencies $\leq 70 \mathrm{MHz}$; thus its flux densities were measured only $\geq 110 \mathrm{MHz}$ [where the signal-to-noise ratio is $\gtrsim 9$; see Fig. 8(b)].

As Fornax A and Pictor A are unresolved at all frequencies, their peak flux densities were extracted using the Miriad task IMFIT. To improve the accuracy of these measurements, we used a two component fit: a Gaussian with the size of the point spread function and an initial offset estimate, in a square region around each source adequately chosen depending on the frequency. The reference flux density values of Fornax A at each frequency were obtained by extrapolating the value at $189 \mathrm{MHz}$ to the observing frequencies using a single power-law spectrum of spectral index -0.88 (see Ref. 23). Similarly, the reference values of Pictor A were obtained by extrapolating the measurement at $150 \mathrm{MHz}$ with a single power-law spectrum of spectral index -0.76 (see Ref. 25).

Centaurus $\mathrm{A}$ is resolved at frequencies $\geq 110 \mathrm{MHz}$. A zoom around this source at 70 and $320 \mathrm{MHz}$ ( $X X$ polarization) is shown in Fig. 8(a). For a proper comparison with the reference values (extrapolated from the value at $118 \mathrm{MHz}$ using a power law of spectral index -0.70 , given in Ref. 21), we thus obtained its integrated flux densities at those frequencies using the AEGEAN source finder tool (see Refs. 26 and 27), within islands (i.e., contiguous groups of pixels that are above a given threshold ${ }^{26}$ ) that we ensured were covering the source extended emission down to the $3 \sigma_{\text {rms }}$ level in each analyzed image (see Ref. 27, their figure 9, for an example image of island extent and location). As AEGEAN does not provide the uncertainties on the island flux densities (see Refs. 26 and 27), these were estimated as $\sigma_{S}=\sigma_{\text {rms }} \times \sqrt{N_{\text {beams }}}$, with $\sigma_{\text {rms }}$ being the noise level around the source and $N_{\text {beams }}$ the number of beams crossing the source (i.e., the island integration area). Peak flux densities and their uncertainties at frequencies $\leq 70 \mathrm{MHz}$ were obtained in the same way as done for Fornax A and Pictor A, both unresolved at these frequencies. All flux measurements and their uncertainties were finally corrected for the primary beam, taking into account the antenna response both in the direction of the Sun (calibrator) and of each radio source (excluding measurements at $55 \mathrm{MHz}$, already corrected for the primary beam, see Sec. 3.1). The average $\mathrm{EEP}^{5,18}$ per frequency and linear polarization was used in this correction. 
Primary beam corrected fluxes (in Jy) as a function of the LST (in $\mathrm{h}$ ) within $\sim 1 \mathrm{~h}$ across the radio source transit are shown in Fig. 7 for three of the observed frequencies $(55,160$, and $230 \mathrm{MHz}$, from left to right). Figure 7(a) refers to Centaurus A, Fig. 7(b) refers to Fornax A. Measurements of both linear polarizations are shown (red circles and blue squares for $X X$ and $Y Y$, respectively) and compared with the reference flux values (black lines) derived as described above (i.e., extrapolating the measurements given in Refs. 21 and 23 to the respective frequency).

The integrated spectra of Centaurus A (black squares), Fornax A (blue dots), and Pictor A (red diamonds) are shown in Fig. 8(b). Each point in the spectrum (with $1 \sigma_{\text {tot }}$ error bars) is the average of all of the corresponding measurements in the considered LST range and between the two polarizations. The total uncertainty is computed as $\sigma_{\text {tot }}=\sqrt{\left(S \times \sigma_{\text {amp }}\right)^{2}+\left(\sigma_{S}\right)^{2}}$, with $\sigma_{\text {amp }}$ being the amplitude errors on the calibrator, conservatively assumed to be $\sim 5 \%$ (see Refs. 28 and 29), and $\sigma_{S}$ being the uncertainties on the averaged flux density measurements.

This initial analysis shows that, even with a first-order calibration procedure:

1. variations of the measured radio sources flux densities are on the order of $5 \%$ to $10 \%$ for Centaurus A and 5\% to 20\% for Fornax A across the selected LST intervals at most of the frequencies (Fig. 7). These variations are within flux measurements uncertainties;

2. measured flux densities of Centaurus A, Fornax A, and Pictor A are generally consistent with the respective reference values (Figs. 7 and 8);

3. the measured spectra of Pictor A, Fornax A, and Centaurus A across the SKA1-Low bandwidth follow reasonably well the expected power laws extrapolated from the literature (Fig. 8).

Although the match between observations and predictions can be improved, these results offer a quantitative statement of the system stability and the impact of the mutual coupling effects among antennas, i.e., likely up to $20 \%$.

\subsection{Sensitivity}

In this section, we describe the analysis performed to estimate the SKA1-Low sensitivity through AAVS2 observations, using the difference imaging technique. This method has been successfully applied to estimate the sensitivity of the AAVS1 prototype station ${ }^{3}$ and for a preliminary verification of AAVS2 sensitivity (using mostly the same data as in this paper, see Ref. 11, their section II).

\subsubsection{Difference imaging}

We used the same observations described in Sec. 3 (see Table 1) to derive sensitivities at 55, $70,110,160,230$, and $320 \mathrm{MHz}$. The difference imaging method is based on the generation and analysis of the differences between each pair of close-in-time images, over which the sky and the calibration do not appreciably change. The image differences should thus, ideally, include just noise (all astrophysical radio sources and calibration artifacts cancel out, see Refs. 3 and 11). The difference between the two $0.14 \mathrm{~s}$ integration dirty images (Sec. 3) for each snapshot and each polarization were produced through the MATHS task. The rms of the noise in all image differences was measured through the IMHIST task within three square boxes of increasing sizes $(41 \times 41,61 \times 61$, and $81 \times 81$ pixels, respectively) centered around zenith.

\subsubsection{Sensitivity measurements across LST}

For each coarse channel observation, visual inspection of all difference images produced showed that they are mostly noise-like and free of residual emission. Example difference images for the calibration snapshot (Sun transit) at $160 \mathrm{MHz}$ are reported and described in Figs. 12 and 13, Appendix B. A limited number of difference images showed either significant artifacts due to interference from satellite emission crossing the sky (see Sec. 3) or the presence of strong residual emission due to different calibration errors occurring between the two consecutive 
Table 4 Averages of primary beam corrected $r$ sm noise values, measured in difference images within the largest zenith-centered box, as a function of frequency.

\begin{tabular}{lc}
\hline \hline$\nu(\mathrm{MHz})$ & $\left\langle\sigma_{0-24 h}\right\rangle\left(\mathrm{Jybeam}^{-1}\right)$ \\
\hline 55 & 95 \\
70 & 100 \\
110 & 25 \\
160 & 15 \\
230 & 20 \\
320 & 15 \\
\hline \hline
\end{tabular}

integrations. These issues affected $<2 \%$ of the observations at $70,110,230$, and $320 \mathrm{MHz}$, and the corresponding images were discarded from the sensitivity analysis. The three values of the rms of the noise extracted from the zenith-centered square boxes in each difference image were averaged, obtaining one measurement per linear polarization: $\sigma_{p}$ (with $p$ indicating the linear polarization $X X, Y Y$ ). As in the calibration, we did not correct the model flux densities of the Sun for the antenna response (Sec. 3); we applied the primary beam corrections to the $\sigma_{p}$ measurements by multiplying those for the antenna response in the direction of the Sun (normalized to zenith). We used the average $\operatorname{EEP}^{5,18}$ corresponding to each central frequency and linear polarization. At $55 \mathrm{MHz}$, these measurements (already corrected for the primary beam) were re-scaled to the flux density scale, as described in Sec. 3.1. As a reference, we report the measured primary beam corrected rms noise averaged over both polarizations and in the entire 0 to $24 \mathrm{~h}$ range in Table 4.

Each primary beam corrected $\sigma_{p}$ value was converted into the station system equivalent flux density (SEFD) according to the following equation:

$$
\operatorname{SEFD}_{s, p} \approx \frac{\sigma_{p}}{\sqrt{2}} \eta \sqrt{t_{i} B} \frac{N}{256} \mathrm{Jy}
$$

where $p$ indicates the linear polarization $(X X, Y Y)$ and $N$ is the number of used station antennas in the observations; thus the fraction $N / 256$ is a first-order correction for the fact that 6 and 12 antennas were flagged out from the April 2020 data and February 2021 data, respectively (see Sec. 3). Moreover, $t_{i} \approx 0.14 \mathrm{~s}$ is the integration time of each input image (assumed to be the same for all datasets), $B \approx 0.78 \mathrm{MHz}$ is the effective coarse channel bandwidth of each dataset (after the edge fine channels flagging, Sec. 3), and $\eta$ is the system efficiency (that takes into account the finite correlator efficiency and other forms of incoherence ${ }^{1}$ ), assumed to be equal to 1 . The factor $\sqrt{2}$ results from the assumption that the two input images used to make the image difference have identical Gaussian noise characteristics; thus the rms of the image difference is a factor $\sqrt{2}$ of the actual noise of a single snapshot image. Note moreover that the total intensity is defined as $I=\left(I_{X X}+I_{Y Y}\right) / 2$. Equation (2) is then used to compute the measured station sensitivity

$$
s_{s, p} \equiv \frac{A_{\mathrm{eff}}}{T_{\mathrm{sys}}}=10^{26} \frac{2 k}{\mathrm{SEFD}_{s, p}} \mathrm{~m}^{2} \mathrm{~K}^{-1}
$$

where $k$ is the Boltzmann constant $\left(k=1.3810^{-23} \mathrm{~m}^{2} \mathrm{~kg} \mathrm{~s}^{-2} \mathrm{~K}^{-1}\right), A_{\text {eff }}$ is the effective area of the station, and $T_{\text {sys }}$ is the system temperature. The estimated SEFD of the entire SKA1-Low array, to a first-order approximation for the large number of SKA1-Low stations (512), is thus simply 


$$
\mathrm{SEFD}_{\mathrm{SKA}, p} \approx \frac{\mathrm{SEFD}_{s, p}}{512} \mathrm{Jy} \text {. }
$$

The measured SKA1-Low sensitivity was thus given as

$$
s_{\mathrm{SKA}, p} \equiv \frac{A_{\text {eff }}}{T_{\mathrm{sys}}}=10^{26} \frac{2 k}{\mathrm{SEFD}_{\mathrm{SKA}, p}} \mathrm{~m}^{2} \mathrm{~K}^{-1} .
$$

The estimated SKA1-Low sensitivity curves as a function of LST across the full length of the observation ( 22 to $24 \mathrm{~h}$ ) were derived at all analyzed frequencies. An example of these curves at $160 \mathrm{MHz}$ is shown in Figs. 9(a) and 9(b), for the two linear polarizations. The corresponding
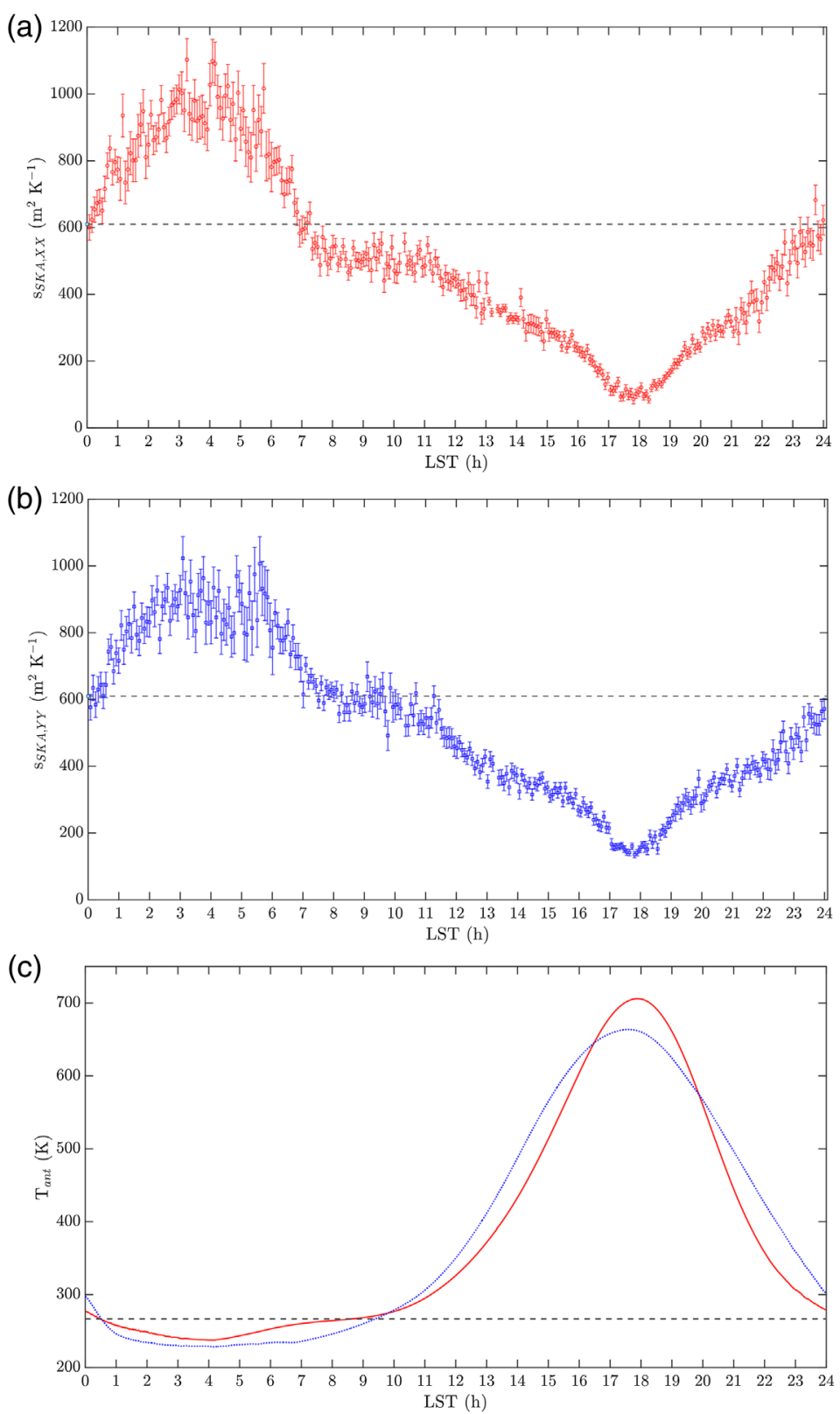

Fig. 9 (a, b) 2020/04/07-08, $160 \mathrm{MHz} s_{S K A, p}$ sensitivity (in $\mathrm{m}^{2} \mathrm{~K}^{-1}$ ) as a function of LST (in $\mathrm{h}$ ), compared with the SKA1-Low sensitivity requirement (dashed line ${ }^{30}$ ); red circles and blue squares are estimates for $X X$ and $Y Y$ polarizations, respectively, with $1 \sigma$ error bars. (c) Corresponding $T_{\text {ant }}$ (in K) simulation as a function of LST for the same date and time range; red solid and blue dotted lines refer to $X X$ and $Y Y$ polarization, respectively. The dashed line is the $T_{\text {sky }}$ value used to compute the SKA1-Low sensitivity requirements. ${ }^{30}$ 
SKA1-Low sensitivity requirement ${ }^{30}$ is also shown by the dashed line. The uncertainties $(1 \sigma$ error bars in the plots) are estimated for every $1 \mathrm{~h}$ interval as the standard deviation of the individual sensitivity measurements, after subtraction of the baseline polynomial fit of the full $24 \mathrm{~h}$ data. As expected, the measured sensitivity changes as a function of time because the sky temperature varies with time due to the rise and set of the Galactic plane and the Galactic center (see Refs. 7, 11, and 31). The highest sensitivity values are reached when the emission is below the horizon and the Sun is close to its transit [LST 3 to $4 \mathrm{~h}$ at $160 \mathrm{MHz}$, in Figs. 9(a) and 9(b)]; the sensitivity then decreases as the Galactic plane and center rise and move across the field of view, reaching its minimum value at the Galactic center's transit above the array [occurring at $\approx 18 \mathrm{~h}$ LST in the period of our observations, see Figs. 9(a) and 9(b)]. This is evident in the plot of antenna temperature as a function of LST [see Fig. 9(c)], estimated as

$$
T_{\text {ant }}(\nu)=\frac{\int_{4 \pi} P_{p}(\nu, \theta, \phi) T(\nu, \theta, \phi) \mathrm{d} \Omega}{\int_{4 \pi} P_{p}(\nu, \theta, \phi) \mathrm{d} \Omega},
$$

where $P_{p}(\nu, \theta, \phi)$ is the average EEP (per polarization, $\left.{ }^{5,18}\right)$ and $T(\nu, \theta, \phi)$ is the sky brightness temperature at frequency $\nu$ and pointing direction $(\theta, \phi)$ simulated as described in Sec. 3. We take this into account when comparing with the sensitivity requirements (Sec. 4.2.3).

The sensitivity estimates for the two linear polarizations, averaged over the entirety of the LST ranges, are consistent with each other within $\lesssim 15 \%$ at all analyzed frequencies except at $70 \mathrm{MHz}$, where $X X$ values are a factor of $\sim 2$ higher with respect to $Y Y$ estimates. This discrepancy might be related to mutual coupling effects (see Refs. 5 and 18); however, it needs to be further investigated.

We note that, even if our calibration procedure takes into account the fact that the calibrator source is not at zenith (Sec. 3), our sensitivity measurements most likely underestimate the actual SKA1-Low zenith sensitivity that would be reachable through an ideal calibration leading to thermal noise.

For frequencies $\geq 70 \mathrm{MHz}$, self calibration with the Sun was also applied by selecting subsets of snapshots in the LST ranges corresponding to Sun elevations $\geq+45^{\circ}$, to avoid calibration inaccuracies related to the "naturally" degraded sensitivity of the antenna at low elevations. ${ }^{24}$ The sensitivities derived through self calibration are consistent with those obtained through calibration using a single snapshot at the Sun transit (in the common LST intervals) for all frequencies $\leq 160 \mathrm{MHz}$, suggesting a good system calibration stability over several hours (see also Ref. 6). However, at frequencies $\geq 230 \mathrm{MHz}$, we found offsets between the two sensitivity estimates, with single snapshot calibration providing systematically lower values with respect to self calibration. As self calibration removes the time dependencies of the system, we expect such sensitivity estimates to be more accurate. For this reason, the sensitivity measurements at 230 and $320 \mathrm{MHz}$ obtained with single snapshot calibration across the full length of the observations were consistently re-scaled (as detailed in Pupillo et al. 2020, internal SKA Observatory progress report, available on request).

\subsubsection{Comparison with sensitivity simulations and requirements}

In this section, we present a comparison of our experimental estimates of the SKA1-Low sensitivity with the requirements and sensitivities derived from electromagnetic (EM) simulations across the entire bandwidth (50 to $350 \mathrm{MHz}$ ).

SKA1-Low sensitivity requirements are specified at each frequency $\nu$ (in $\mathrm{GHz}$ ) for a uniform sky temperature model, computed using the following formula (see Refs. 1 and 30, their page 283):

$$
T_{\text {sky,r }}(\nu) \approx 20\left(\frac{0.408}{\nu}\right)^{2.75}+2.73 \mathrm{~K}
$$

where the first term is the extrapolation of a the temperature typical of a cold sky patch in the Haslam map ${ }^{32}$ and the second term is the CMB temperature. Requirements thus do not consider the sky temperature variations over the $24 \mathrm{~h}$ due to the spatial variations of the Galactic emission 
Table 5 Estimated SKA1-Low sensitivities as a function of frequency, averaged between $X X$ and $Y Y$ polarizations, compared with the sensitivity requirements $\left(s_{\mathrm{SKA}, \mathrm{r}}\right)$ : mean sensitivities are presented over the LST interval where the Galactic center is below the horizon (second column) and over the $24 \mathrm{~h}$ interval (third column).

\begin{tabular}{lccc}
\hline \hline$\nu(\mathrm{MHz})$ & $\left\langle s_{\mathrm{SKA}}\right\rangle_{0-8 \mathrm{~h}}\left(\mathrm{~m}^{2} \mathrm{~K}^{-1}\right)$ & $\left\langle s_{\mathrm{SKA}}\right\rangle_{0-24 \mathrm{~h}}\left(\mathrm{~m}^{2} \mathrm{~K}^{-1}\right)$ & $s_{\mathrm{SKA}, \mathrm{r}}{ }^{30}\left(\mathrm{~m}^{2} \mathrm{~K}^{-1}\right)$ \\
\hline 55 & 150 & 110 & 70 \\
70 & 210 & 120 & 140 \\
110 & 630 & 390 & 530 \\
160 & 810 & 520 & 610 \\
230 & 700 & 500 & 610 \\
320 & 810 & 630 & 550 \\
\hline \hline
\end{tabular}

[see Fig. 9(c) and Sec. 4.2.3]. From Eq. (7), the sensitivity requirement at $160 \mathrm{MHz}$ is $T_{\mathrm{sky}, \mathrm{r}}=$ $267 \mathrm{~K}$ [see also Fig. 9(c), dashed line]. Our $T_{\text {ant }}$ simulation at this frequency [see Sec. 4.2 and Fig. 9 (c), red and blue lines] is closer to $T_{\text {sky,r }}$ between $\approx 0 \mathrm{~h}$ and $\approx 10 \mathrm{~h}$ LST. Therefore, we chose the 0 to $8 \mathrm{~h}$ LST range as the appropriate interval to compare sensitivity estimates and requirements. However, we add the corresponding mean sensitivity estimates within the entire $24 \mathrm{~h}$ LST range for completeness (Table 5).

Results are shown in Fig. 10; black squares are the 0 to $8 \mathrm{~h}$ LST SKA1-Low mean sensitivity estimates (in $\mathrm{m}^{2} \mathrm{~K}^{-1}$ ) as a function of frequency (in MHz; see Table 5). Error bars are included within the symbol size and correspond to $1 \sigma$ standard deviations of the averaged values, i.e., the square root of the variance of the average over the LST interval 0 to $8 \mathrm{~h}$ and two polarizations.

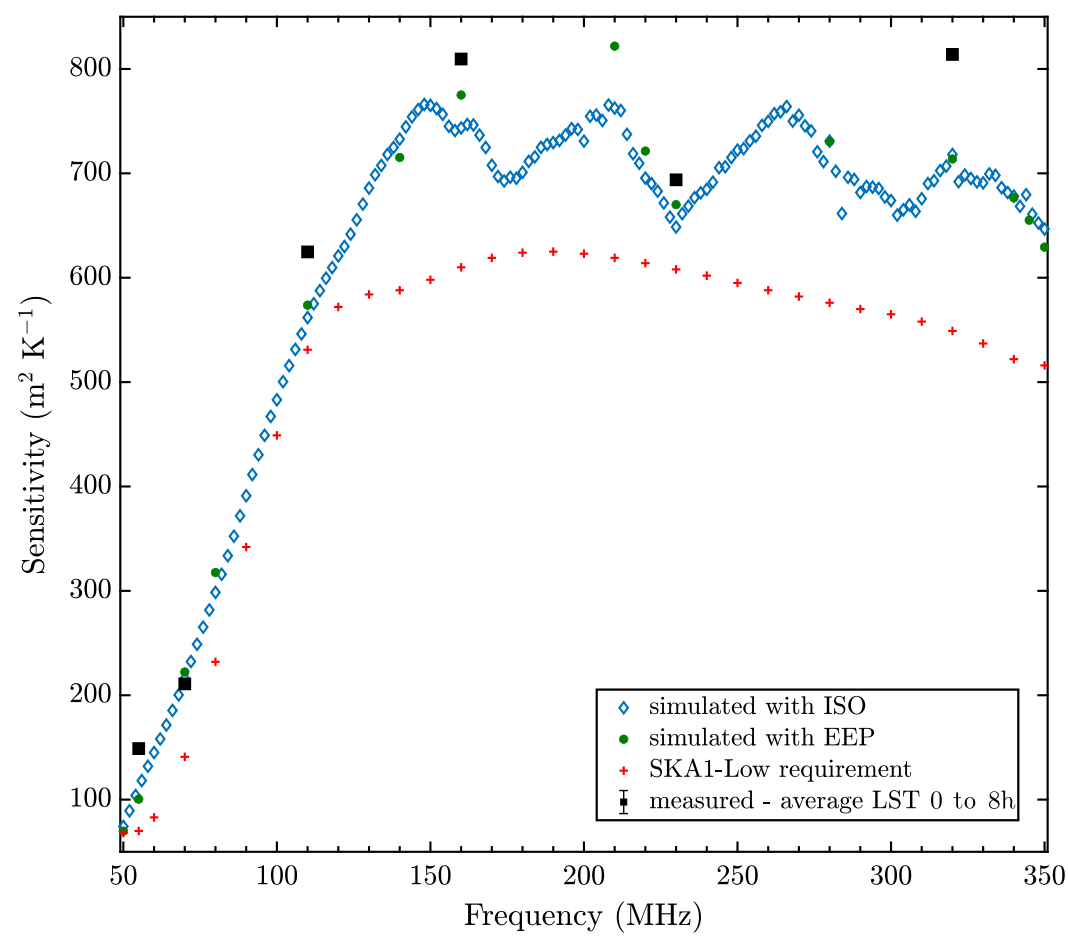

Fig. 10 Zenith sensitivity over the SKA1-Low bandwidth (averages of $X X$ and $Y Y$ polarizations). Black squares are the mean sensitivity estimates in LST 0 to $8 \mathrm{~h}$ (Table 5), with $1 \sigma$ error bars within the symbol size (Sec. 4.2). Blue diamonds and green circles are the simulated sensitivities computed using the isolated SKALA4.1 antenna patterns and the EEP, respectively. Red crosses are the SKA1-Low requirements ${ }^{30}$ (see Table 5). 
Note that these do not include the uncertainties on the absolute flux scale, assumed to be $~ 5 \%$ (see also Sec. 4.1). These estimates are compared with EM simulations of sensitivity at zenith for one station ${ }^{18}$ and here are re-scaled for the whole SKA1-Low; blue diamonds result from the sensitivity computed from the isolated SKALA4.1 pattern (therefore without mutual coupling effects), whereas green circles are from the EEP, and both of them are averages of the $X X$ and $Y Y$ polarizations. Overall, the agreement between the two simulated sensitivities at zenith is very good, meaning that the mutual coupling does not deteriorate the sensitivity (see Refs. 5 and 18). The simulation of sensitivity from the isolated patterns has been executed for frequencies in the range 50 to $350 \mathrm{MHz}$, with a step of $2 \mathrm{MHz}$. The simulation from the EEP was performed at 50, $55,70,80,110,140,160,210,220,230,280,320,340,345$, and $350 \mathrm{MHz}$. The requirements are shown with the red crosses. As stated in Sec. 4.2, our measurements are more likely underestimates of the actual SKA1-Low zenith sensitivity, which would be reachable in ideal, thermal noise limited images. Moreover, our estimates are averages in LST 0 to $8 \mathrm{~h}$, thus not exactly comparable with these simulations. However, we find an overall good agreement for most of the analyzed frequencies, with measured averages (black squares in Fig. 10) generally higher than the EEP simulated sensitivity (green circles in Fig. 10), with differences that range between $\sim 3.5 \%$ (at $230 \mathrm{MHz}$ ) and $\sim 13 \%$ (at $320 \mathrm{MHz}$ ). Finally, all our estimates in LST 0 to $8 \mathrm{~h}$ exceed the SKA1-Low requirements (red crosses in Fig. 10) by factors ranging from $\sim 1.2$ (at $70 \mathrm{MHz}$ ) to $\sim 2.3$ (at $55 \mathrm{MHz}$ ).

\section{Conclusion and Future Work}

The work presented in this paper provides an initial characterization of the SKA1-Low prototype station AAVS2 performance, such as calibratability and sensitivity. We used commissioning AAVS2 snapshot observations at six different frequencies, from 55 to $320 \mathrm{MHz}$, selected to sample the SKA1-Low bandwidth.

We verified the array calibratability and all-sky imaging capabilities of the station (used as a stand-alone interferometer) with simple Sun-based calibration, obtained using the Sun as a pointlike calibration source at its transit and transferring the solutions to 22 to $24 \mathrm{~h}$ snapshots data collected every $5 \mathrm{~min}$ (Sec. 3). The achieved good quality of images confirms calibration and system stability over timescales of $24 \mathrm{~h}$ (Sec. 3). Our initial consistency checks on selected radio sources flux densities also corroborate this finding, as they show $\lesssim 20 \%$ variations $\approx 1 \mathrm{~h}$ across their transit (Sec. 4.1), which can be considered acceptable as they are within the flux measurements uncertainties. Moreover, the quality of absolute flux scales derived through first-order calibration methods is relatively good, with integrated spectra of Centaurus A, Fornax A, and Pictor A following quite well the expected power-laws extrapolated from the literature measurements (Sec. 4.1).

We also derived "zenith" sensitivity estimates through the difference imaging technique (Sec. 4.2). For this analysis, self calibration during daytime, with elevation of the Sun $\geq 45^{\circ}$, was also applied (Sec. 3). The comparison between self and single snapshot calibration sensitivities shows that they are consistent with each other at frequencies $\leq 160 \mathrm{MHz}$ (see Sec. 3), confirming that the system calibration is stable over several hours. Another important result of this work is that our sensitivity estimates, averaged between the two linear polarizations and in LST range 0 to $8 \mathrm{~h}$ (corresponding to the "cold" sky patch and where $T_{\text {ant }}(\nu)$ is closer to the uniform $T_{\text {sky,r }}(\nu)$ used to compute the SKA1-Low specifications, Sec. 4.2.3) are from $\sim 1.2$ to $\sim 2.3$ times the corresponding SKA1-Low sensitivity requirements. Moreover, they are in good agreement with the EM sensitivity simulations (differences $\lesssim 13 \%$, Sec. 4.2.3).

For future work, we plan to extend this analysis using additional commissioning observations and observing frequencies, both already available and to be performed performed as long as AAVS2 station remain operational. Different calibration methods for the lowest frequencies $(\leq 70 \mathrm{MHz}$ ), such as self calibration in the night-time LST ranges using a set of model bright sources in the observed field of view or against an all-sky model for the diffuse emission (with the Sun added), will allow for improving the accuracy of data calibration, imaging, and sensitivity analysis. Moreover, we plan to use our sky temperature simulations (Sec. 4.2.3) to provide estimates of the SKA1-Low sensitivity as a function of $T_{\text {sky }}$ variability. All of this will be presented in future publications. 
Remarkably, the work presented here is an important step forward in the SKA-Low project toward construction as the roll out of the telescope is approaching. The construction of the production prototype Aperture Array 0.5 (AA0.5), consisting of six full SKA-Low stations, such as AAVS2, is expected to start in the early $2023 .{ }^{6}$ Hence, an analysis similar to the one presented here will be extended to AA0.5 interferometric observations to verify its performance.

\section{Appendix A: Embedded Element Patterns}

The averages of the EEPs as a function of zenith angle, in the $H$ and $E$ plane for $Y$ polarization, are shown in Fig. 11. These are the primary beams used throughout this paper (see Sec. 3). Note that the corresponding patterns for the $X$ polarization are almost identical. ${ }^{18}$
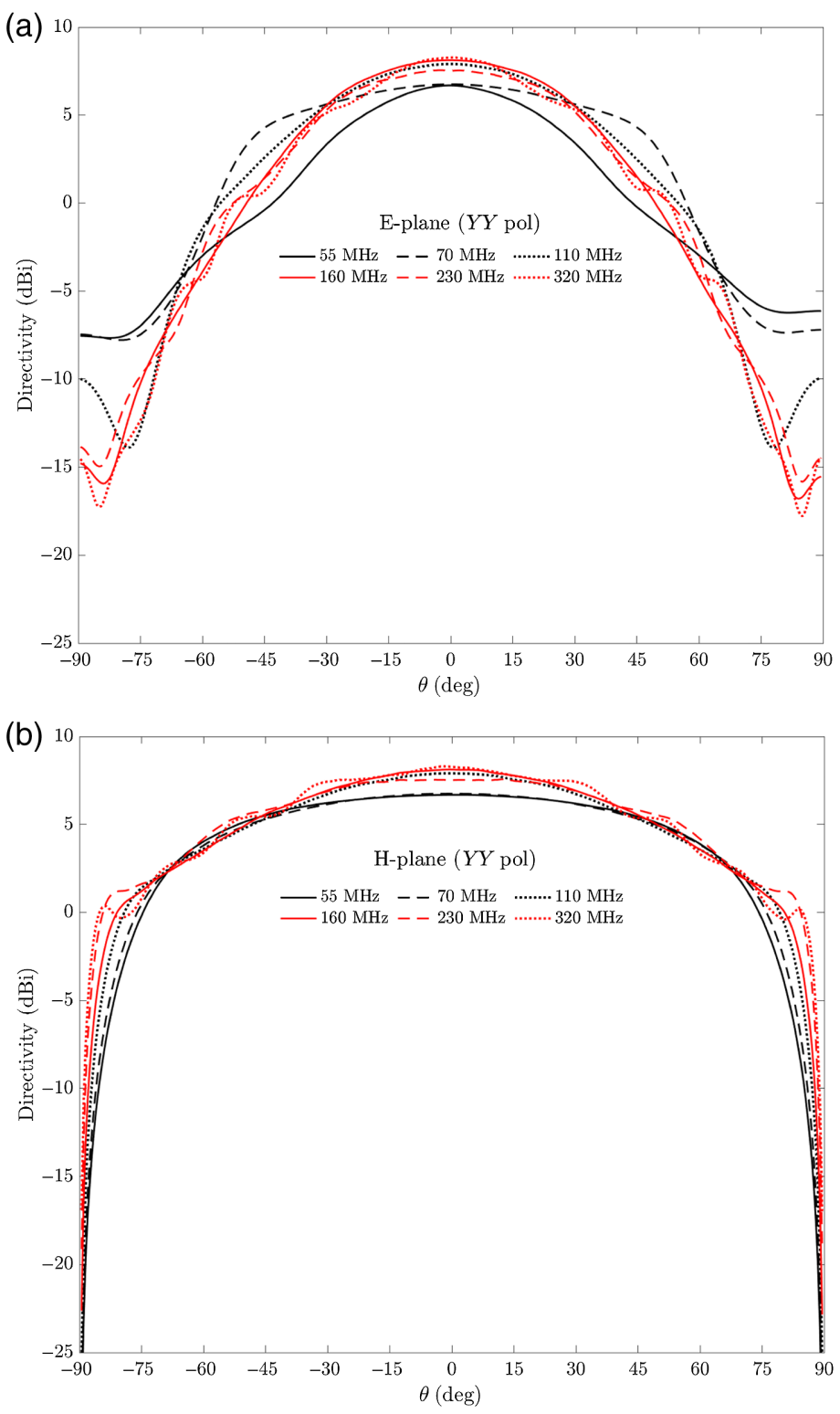

Fig. 11 Co-polar directivities (averages of EEPs) as a function of zenith angle for the 256 antennas comprising AAVS2. The excited port is aligned to the $Y$-axis. (a) $E$-plane; (b) $H$-plane. Each plot shows all six frequencies analyzed in this work: $55 \mathrm{MHz}$ (black solid), $70 \mathrm{MHz}$ (black dashed), $110 \mathrm{MHz}$ (black dotted), $160 \mathrm{MHz}$ (red solid), $230 \mathrm{MHz}$ (red dashed), and $320 \mathrm{MHz}$ (red dotted). 


\section{Appendix B: Example Difference Images and Noise Distribution at Zenith}

Figures 12 and 13 provide illustrative examples of the difference imaging technique used in this work (see Sec. 4.2.1). Both figures refer to the calibration snapshot (Sun transit) at $160 \mathrm{MHz}(X X$ pol in Fig. 12 and $Y Y$ pol in Fig. 13). Figures 12(a) and 13(a) show the all-sky dirty images derived from the two consecutive $0.14 \mathrm{~s}$ integrations; their difference is reported in Figures 12 (b) and 13(b) (left), where the red circle indicates the horizon and the green zenith centered square is the largest box used for the noise measurements. Note that, in this example snapshot, the Sun is the dominant source in the field of view and no residuals are visible at its position in the difference images. Figures 12(b) and 13(b) (right) show the corresponding histogram distributions of the $\sigma_{p}$ noise (in Jy beam ${ }^{-1}$ ) within the zenith centered box, overlaid with the 1-term best Gaussian model fit (red line). The residual plots are also shown below. The fits are very good for both polarizations $\left(R^{2}=0.9736\right.$ for $\sigma_{X X}$ and $R^{2}=0.9731$ for $\left.\sigma_{Y Y}\right)$. We note that the pixel distribution of the residual image closely follows a Gaussian function, with no noticeable outliers

(a)

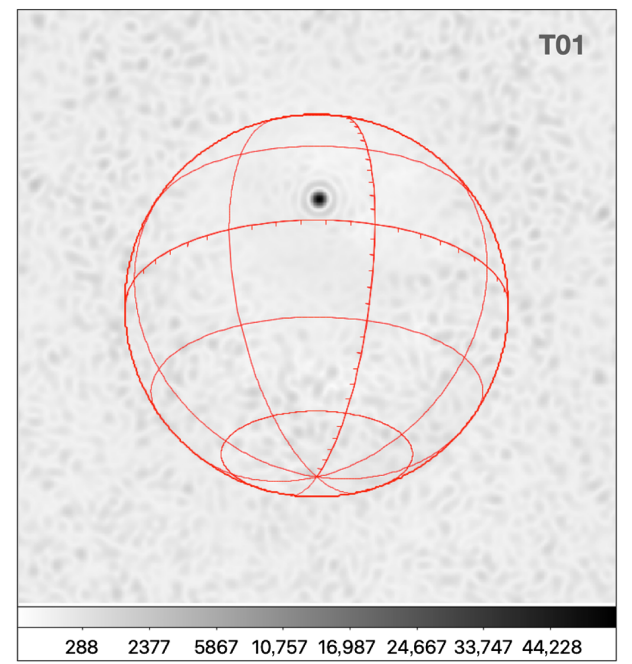

(b)

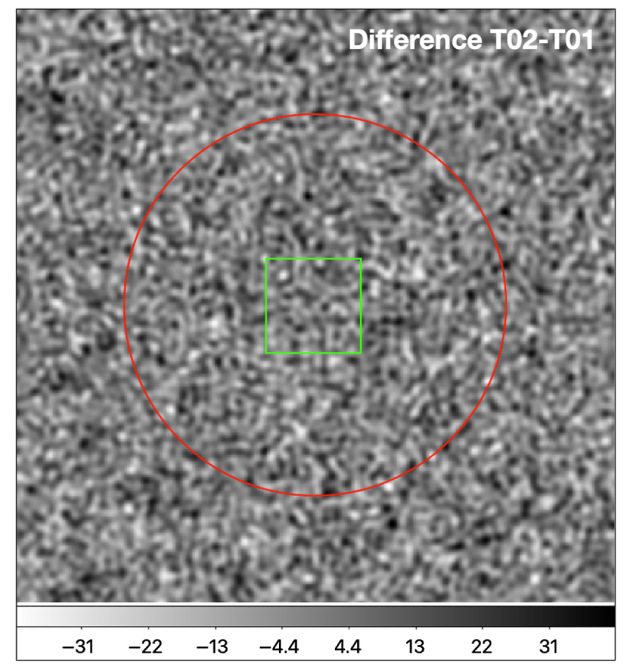

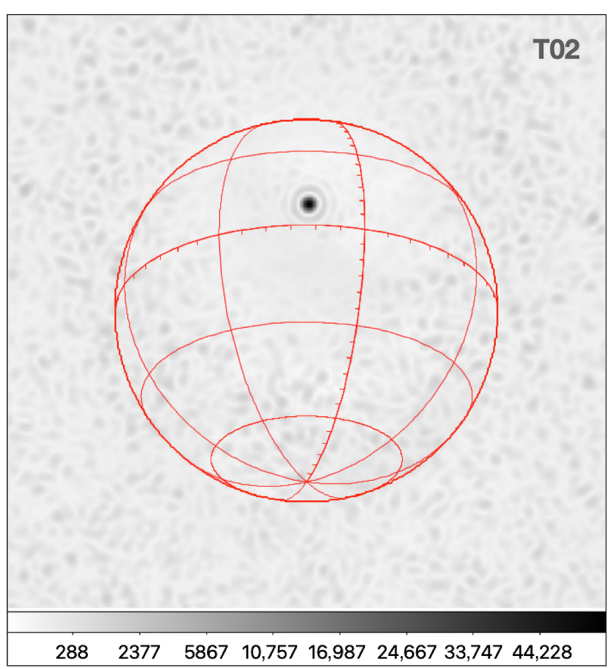
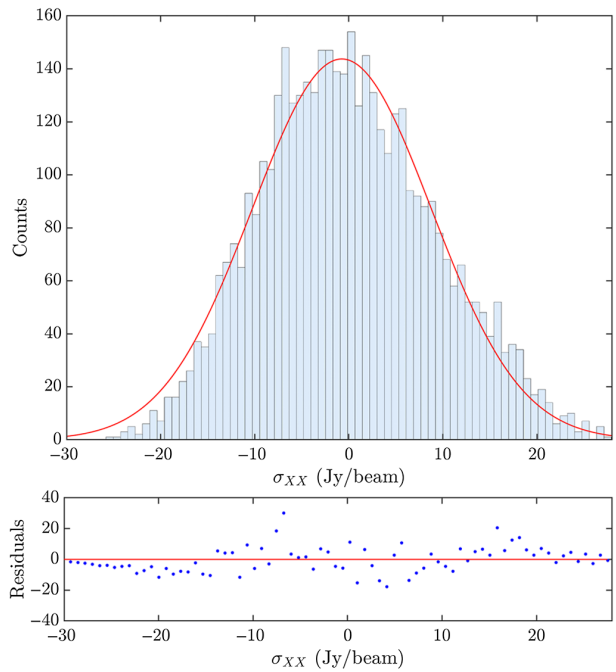

Fig. 12 Example of difference imaging procedure for the calibration snapshot (Sun transit) at $160 \mathrm{MHz}, X X$ polarization. (a) All-sky dirty images for the first and second $0.14 \mathrm{~s}$ consecutive integrations (left and right, respectively). The gray scales (linear, Jy beam ${ }^{-1}$ ) are in the same range. (b) Left is the corresponding difference image. The green square indicates the largest $(81 \times 81$ pixels $)$ box where $\sigma_{p}$ noise is measured. Note the different flux scale with respect to (a). (b) Right is the corresponding histogram distribution of the noise $\sigma_{p}$ within that box with the Gaussian fit overlaid (red curve), along with the residuals (lower plot). 
(a)

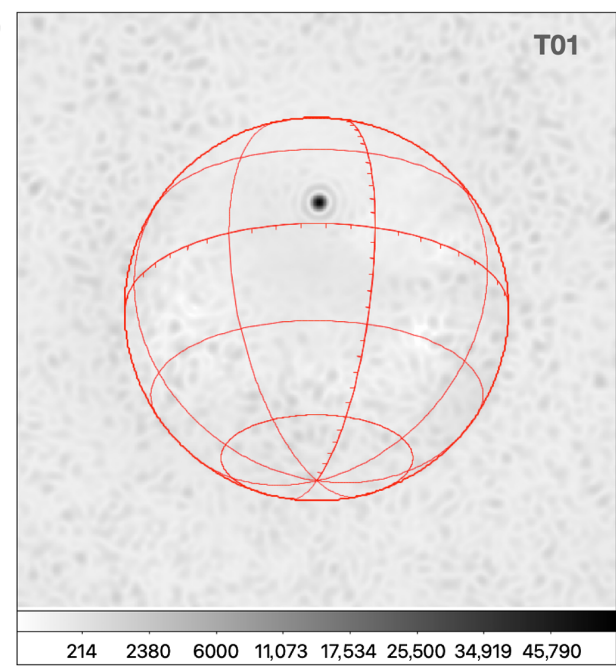

(b)

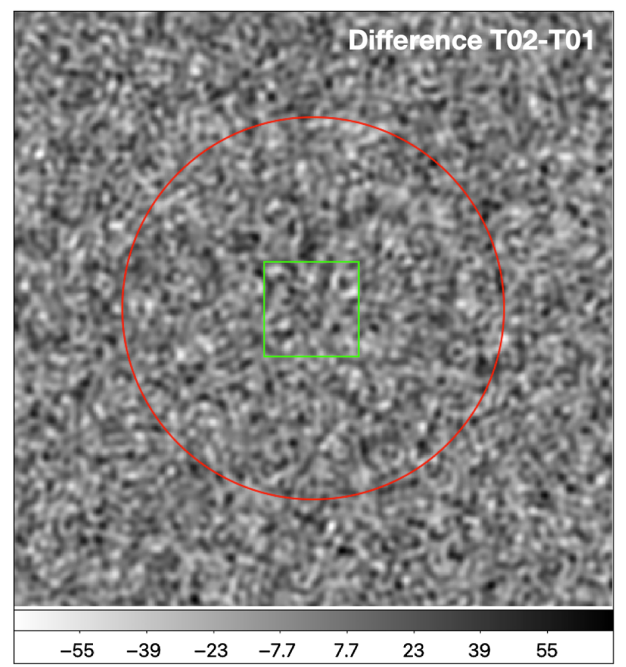

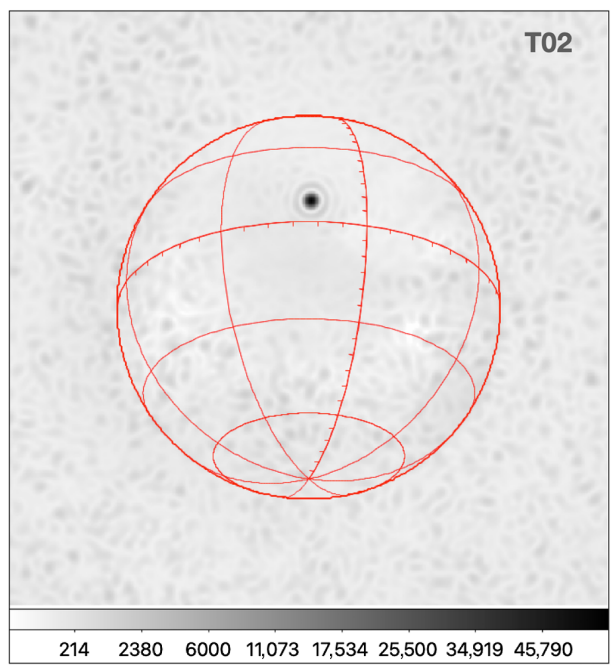
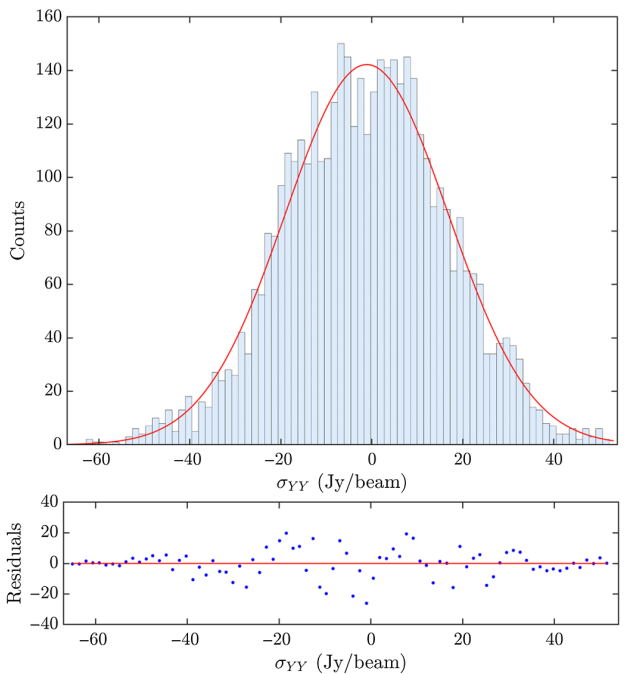

Fig. 13 Same as Fig. 12, for $Y Y$ polarization.

or zero-mean residuals. Even residual artifacts that appear in some snapshots when the Galactic center transits do not significantly affect the Gaussian best fit.

\section{Acknowledgments}

AAVS2 is hosted by the MWA under an agreement via the MWA External Instruments Policy. This work makes use of the MRO, operated by CSIRO. We acknowledge the Wajarri Yamatji people as the traditional owners of the Observatory site. We thank the anonymous referees for their helpful comments.

\section{References}

1. R. Braun et al., "Anticipated performance of the Square Kilometre Array-Phase 1 (SKA1)," Square Kilometre Array Memo, arXiv:1912.12699 (2019).

2. P. E. Dewdney, on behalf of SKAO, SKA1 Design Baseline Description, SKA-TEL-SKO000107 (2020).

3. P. Benthem et al., "The Aperture Array Verification System 1: system overview and early commissioning results," A\&A 655 (2021).

4. R. Wayth et al., "The Engineering Development Array: a low frequency radio telescope utilising SKA precursor technology," Publ. Astron. Soc. Aust. 34, e034 (2017). 
5. D. B. Davidson et al., "Electromagnetic modelling of the SKA-Low AAVS2 prototype," in XXXIIIrd Gen. Assembly and Sci. Symp. Int. Union of Radio Sci., pp. 1-4 (2020).

6. A. J. J. van Es et al., "A prototype model for evaluating SKA-Low station calibration," Proc. SPIE 11445, 1144589 (2020).

7. R. Wayth et al., "The Engineering Development Array 2: design, performance and lessons from an SKA-Low prototype station," J. Astronom. Telesc. Instrum. Syst. 8(1), 011010 (2021).

8. P. Bolli, on behalf of SKAO, Antenna \& Station Performance Update for SKALA4.1-AL, SKA-TEL-SKO-0001099, rev. 01 (2020).

9. M. Sokolowski et al., "A Southern-Hemisphere all-sky radio transient monitor for SKA-Low prototype stations," Publ. Astron. Soc. Aust. 38, e023 (2021).

10. F. Perini et al., "RF over fibre technology for SKA-Low receiver," J. Astronom. Telesc. Instrum. Syst. 8(1) (2022).

11. M. Sokolowski et al., "Preliminary sensitivity verification of the SKA-Low AAVS2 prototype," in 15th Eur. Conf. Antennas and Propag. (EuCAP), pp. 1-5 (2021).

12. S. J. Tingay et al., "A survey of spatially and temporally resolved radio frequency interference in the FM band at the Murchison Radio-astronomy Observatory," Publ. Astron. Soc. Aust. 37, e039 (2020).

13. M. A. Clark, P. C. La Plante, and L. J. Greenhill, "Accelerating radio astronomy crosscorrelation with graphics processing units," Int. J. High Perform. Comput. Appl. 27(2), 178-192 (2013).

14. E. W. Greisen, “AIPS FITS file format," AIPS Memo 117 (2017).

15. R. J. Sault, P. J. Teuben, and M. C. H. Wright, "A retrospective view of MIRIAD," Astron. Soc. Pac. Conf. Ser. 77, 433 (1995).

16. https://www.solarmonitor.org/index.php.

17. A. O. Benz, "4.1.1.6 radio emission of the quiet sun: Datasheet from Landolt-Börnstein group vi astronomy and astrophysics. volume $4 \mathrm{~b}$ : "solar system" in springermaterials (https://doi.org/10.1007/978-3-540-88055-4_5)," Copyright 2009 Springer-Verlag Berlin Heidelberg, Part of Springer Materials (accessed 23 March 2021).

18. P. Bolli et al., "Computational electromagnetic simulation of the SKA-Low prototype station AAVS2," JATIS, SKA Observatory Special Section, under review (December 2021).

19. A. de Oliveira-Costa et al., "A model of diffuse Galactic radio emission from $10 \mathrm{MHz}$ to $100 \mathrm{GHz}$, "Mon. Not. R. Astron. Soc. 388, 247-260 (2008).

20. https://github.com/telegraphic/PyGSM

21. B. McKinley et al., "The giant lobes of Centaurus A observed at $118 \mathrm{MHz}$ with the Murchison Widefield Array," Mon. Not. R. Astron. Soc. 436, 1286-1301 (2013).

22. G. Comoretto and S. Chiarucci, "SKA project series-quantization effects and nonlinearities in multi-bit correlation," Arcetri Technical Reports, 2018, https://openaccess.inaf.it/ handle/20.500.12386/1002.

23. G. Bernardi et al., "A $189 \mathrm{MHz}, 2400 \mathrm{deg} 2$ polarization survey with the Murchison widefield array 32-element prototype," Astrophys. J. 771, 105 (2013).

24. P. Bolli et al., "Test-driven design of an active dual-polarized log-periodic antenna for the Square Kilometre Array," IEEE Open J. Antennas Propag. 1, 253-263 (2020).

25. D. C. Jacobs et al., "A flux scale for Southern Hemisphere $21 \mathrm{~cm}$ Epoch of reionization experiments," Astrophys. J. 776, 108 (2013).

26. P. J. Hancock et al., "Compact continuum source finding for next generation radio surveys," Mon. Not. R. Astron. Soc. 422, 1812-1824 (2012).

27. P. J. Hancock, C. M. Trott, and N. Hurley-Walker, "Source finding in the era of the SKA (Precursors): Aegean 2.0," Publ. Astron. Soc. Aust. 35, e011 (2018).

28. A. M. M. Scaife and G. H. Heald, "A broad-band flux scale for low-frequency radio telescopes," Mon. Not. R. Astron. Soc. 423, L30-L34 (2012).

29. G. Bernardi et al., "Foregrounds for observations of the cosmological $21 \mathrm{~cm}$ line. I. First Westerbork measurements of Galactic emission at $150 \mathrm{MHz}$ in a low latitude field," Astron. Astrophys. 500, 965-979 (2009).

30. M. Caiazzo on behalf of SKAO, SKA Phase 1 System Requirements Specification, SKATEL-SKO-0000008, rev. 11 (2017). 
31. S. J. Wijnholds and W. A. van Cappellen, "In situ antenna performance evaluation of the LOFAR phased array radio telescope," IEEE Trans. Antennas Propag. 59, 1981-1989 (2011).

32. C. G. T. Haslam et al., "A $408 \mathrm{MHz}$ all-sky continuum survey. II. The atlas of contour maps.," Astron. Astrophys. Suppl. Ser. 47, 1-143 (1982).

Giulia Macario is a postdoctoral fellow at INAF Arcetri Astrophyisical Observatory, where, since 2019, she has worked full time on characterizing AAVS2 through commissioning observations, as a member of the INAF SKA-Low calibration group. She received her $\mathrm{PhD}$ in astrophysics from the University of Bologna, Italy, in 2011. Her main research interest has been related to observational studies of diffuse radio emission in galaxy clusters at low frequencies. She has also been a member of the LOFAR Surveys Key Science Project, contributing to LOFAR commissioning activities.

Giuseppe Pupillo received his MSc (cum laude) and his PhD degrees in astronomy from the University of Bologna, Italy. He is a technology researcher at the INAF-IRA Institute (Bologna), where he is mainly involved in the Square Kilometer Array (SKA) project. His main research interests comprise astronomical low-frequency aperture arrays, observations of fast radio bursts (FRBs) with the northern cross array at Medicina, and radar systems for near-earth objects observations and space surveillance.

Gianni Bernardi received his $\mathrm{PhD}$ from Bologna University in 2004. He is currently a researcher at INAF-IRA and a visiting professor at Rhodes University. He works on observational 21-cm cosmology.

Biographies of the other authors are not available. 